\title{
A Blockchain Research Framework
}

\author{
What We (don't) Know, Where We Go from Here, and How We Will Get There
}

\author{
Marten Risius • Kai Spohrer
}

Received: 11 January 2017/ Accepted: 14 August 2017/Published online: 5 December 2017

(C) US Government 2017

\begin{abstract}
While blockchain technology is commonly considered potentially disruptive in various regards, there is a lack of understanding where and how blockchain technology is effectively applicable and where it has mentionable practical effects. This issue has given rise to critical voices that judge the technology as over-hyped. Against this backdrop, this study adapts an established research framework to structure the insights of the current body of research on blockchain technology, outline the present research scope as well as disregarded topics, and sketch out multidisciplinary research approaches. The framework differentiates three groups of activities (design and features, measurement and value, management and organization) at four levels of analysis (users and society, intermediaries, platforms, firms and industry). The review shows that research has predominantly focused on technological questions of design and features, while neglecting application, value creation, and governance. In order to foster substantial blockchain research that addresses meaningful questions, this study identifies several avenues
\end{abstract}

Accepted after two revisions by the editors of the special issue.

Electronic supplementary material The online version of this article (https://doi.org/10.1007/s12599-017-0506-0) contains supplementary material, which is available to authorized users.

Dr. M. Risius $(\bowtie)$

College of Business and Behavioral Science, Clemson

University, 515 Calhoun Dr, Clemson, SC 29634, USA

e-mail: risius@g.clemson.edu

Dr. K. Spohrer

University of Mannheim, Chair of General Management and

Information Systems, L 15, 1-6-Room 520, 68161 Mannheim, Germany

e-mail: spohrer@uni-mannheim.de for future studies. Given the breadth of open questions, it shows where research can benefit from multidisciplinary collaborations and presents data sources as starting points for empirical investigations.

Keywords Blockchain - Research framework - Literature review $\cdot$ Distributed ledger technology $\cdot$ Digitalization

\section{Introduction}

Blockchain technology currently receives a lot of public attention as advocates argue that it constitutes the foundation for truly trust-free economic transactions based on its unique technological characteristics (Glaser 2017). Blockchain technology is among the most trending technologies (Gartner 2016) and argued to disrupt various intermediary services (Tapscott and Tapscott 2016). It acquired fame as the technology underlying Bitcoin (Beck and Müller-Bloch 2017) but is currently expanded to other areas of application (Wörner et al. 2016).

At the same time, however, scholars are drawing parallels between blockchain technology and, for example, bubble memory regarding their revolutionary impact on business, remembering that bubble memory also never lived up to the expectations associated with it (Avital et al. 2016). Glaser (2017) repeats the commonly expressed concern that blockchain technology is an innovative technology searching for use cases. Despite the great expectations, there is currently a paucity of knowledge regarding where and how blockchain technology is effectively applicable and where it can provide mentionable societal effects. We argue that research can help overcome this paucity by comprehensively understanding the effects of unique blockchain properties (e.g., decentralization, 
transaction speed, security, auditability and control) and by investigating respectively appropriate societal fields of application. So far, however, application-oriented contributions to blockchain research appear to be scarce, disconnected and focused on a limited number of topics (e.g., payment systems). To address these issues, we draw on an established research framework that has previously helped structure and create a meaningful research stream in the related area of social media and business transformation (Aral et al. 2013). We adapt this framework to the particularities of the blockchain technology. By drawing on a fruitfully established framework and transferring the corresponding research questions, we intend to systematically organize findings and develop research topics that look beyond the currently considered subjects. Thereby, we address two research questions: What is the current state of knowledge regarding blockchain, and how can it purposefully be advanced?

To achieve our research objective, we systematically collected published scholarly blockchain papers to review them under consideration of the related research framework and relevant technological foundations (Tschorsch and Scheuermann 2016). In contrast to existing blockchain frameworks (Yli-Huumo et al. 2016), this approach enables us to structure current findings as well as inspire research questions beyond the focus of extant work. Moving forward from the current state of research, we highlight links to other disciplines and propose starting points for empirical research by pointing at some available data sources that can help close the large discrepancy between scholarly knowledge and expectations. To substantiate a long-term contribution, we provide online access to the framework and invite collaborative paper submissions to keep the literature overview up to date. ${ }^{1}$

The remainder of the paper is structured as follows. First, we introduce the key technological concepts underlying blockchain technology. Subsequently, we describe the study's processes of collecting and analyzing the literature before introducing the adapted framework with the respective research questions and existent findings. We then highlight links to related disciplines as several larger questions may require IS researchers to collaborate with scholars of other disciplines or at least consider their theories in order to conduct relevant and rigorous research. Lastly, we present promising data sources for empirical investigations and critically discuss the work's contributions before deriving general conclusions.

\footnotetext{
${ }^{1}$ We provide open access to the overview of current scientific knowledge [Table 2 and Table A1 (in the appendix, available online via http://link.springer.com)] here: http://bit.ly/BCSOTA. We are thankful to Florian Glaser for his inspiring feedback to the avenues for future research.
}

\section{Conceptual Background}

In its generic form, blockchain technology ${ }^{2}$ refers to a fully distributed system for cryptographically capturing and storing a consistent, immutable, linear event log of transactions between networked actors. This is functionally similar to a distributed ledger that is consensually kept, updated, and validated by the parties involved in all the transactions within a network. In such a network, blockchain technology enforces transparency and guarantees eventual, system-wide consensus on the validity of an entire history of transactions. As current blockchain technology can not only process monetary transactions but can also ensure that transactions comply with programmable rules in the form of "smart contracts"(Tschorsch and Scheuermann 2016), it allows even parties who do not fully trust each other to conduct and reliably control mutual transactions without relying on the services of any trusted middlemen. This may be one reason why nearly all banks are currently engaged in developing a vision of what this technology means for their business (Glaser 2017).

Beyond their primary distributed ledger functionality, single implementations of blockchain technology differ in their technical details and capabilities. Recent publicly available blockchains (e.g., Ethereum or Hyperledger Fabric) comprise elements for implicitly managing a fully distributed network of peers, different cryptography-enabled consensus mechanisms for capturing and storing transactions as well as data attached to transactions, and programming languages to create smart contracts of immutable or dynamic business functionality that can be used during transactions (Glaser 2017). Implementations differ regarding their mechanisms to enforce consensus, the power of included programming languages, their capabilities to define who is allowed to participate in a network, and the type of cryptocurrency they include (Beck and Müller-Bloch 2017; Yli-Huumo et al. 2016).

Recent reviews of technical papers on blockchain research show that the majority of scholarly work has focused on improvements and challenges of current protocols, primarily for cryptocurrencies in general and for Bitcoin in particular (Glaser and Bezzenberger 2015; Morisse 2015; Tschorsch and Scheuermann 2016; YliHuumo et al. 2016). While security, data privacy, and usability in these blockchain implementations are subject to ongoing development, particularly the question of the best algorithms to incentivize and ensure transactional validity and consensus is fiercely discussed in research and practice (Walsh et al. 2016). As such, proof-of-work

\footnotetext{
${ }^{2}$ In the following also interchangeably referred to as "blockchain", "blockchain systems", "blockchain environment", or "decentralized blockchain".
} 
approaches that require high levels of energy but guarantee relatively high levels of consistency and protection against forgery by any actor in the network (e.g., in Bitcoin) compete against less costly ones (for a comprehensive introduction see Tschorsch and Scheuermann (2016)). Such alternative approaches require a portion of trust in some elements of the network, such as actors based on the resources they put at risk during validation (e.g., proof-ofstake in Peercoin) or in the manufacturers of devices that are used to validate transactions (e.g., proof-of-elapsedtime in Hyperledger Sawtooth Lake). Blockchain implementations that target the general (not to be trusted) public (e.g., Ethereum, Bitcoin) typically include reward mechanisms based on cryptocurrencies to incentivize actors to verify transactions ("mining Bitcoins") whereas implementations targeting closed, rather trustworthy or at least mutually familiar groups of users (e.g., Hyperledger Fabric) put more emphasis on permissioning mechanisms that allow for granting participation rights to identifiable and accountable actors while denying them to others. In sum, the different approaches towards validation and consensus building aim for different balances regarding availability, consistency, and trustworthiness (Tschorsch and Scheuermann 2016). They thereby influence the potential applications and affordances of each implementation of blockchain technology (Glaser and Bezzenberger 2015). By separating such technical decisions into modular layers that can easily be changed, blockchain technology gains enormous application possibilities beyond simply exchanging tokens of a single cryptocurrency like Bitcoin (Glaser 2017). In fact, some scholars even propose that this technology paves the way for entirely new models of business and organization as it allows for economically reasonable transactions with potentially untrustworthy transaction partners without any additional measures of precaution. They promote the vision of a trust-free economy with truly virtual organizations and automatic business transactions of devices in the internet of things (Beck et al. 2016; Christidis and Devetsikiotis 2016; Glaser 2017; Puschmann and Alt 2016).

Against this backdrop, we believe that it holds merit to throw the spotlight on research that focuses on the wider ramifications of blockchain technology beyond technical details and cryptocurrencies. Prior work fruitfully reviewed and synthesized technical research on protocol improvements, primarily with implications for cryptocurrencies like Bitcoin (Glaser and Bezzenberger 2015; Morisse 2015; Tschorsch and Scheuermann 2016; Yli-Huumo et al. 2016). Yet, little is known about research that delves into the purported disruptive potential of blockchain technology that extends beyond IT (Beck and Müller-Bloch 2017). In light of this broad reach, we strive to structure extant work on blockchain technology beyond cryptocurrencies and aim to provide a conceptual framework that outlines a research agenda with guidelines for researchers from IS as well as from neighboring disciplines.

\section{Method for Structuring Blockchain Advancements}

This study intends to provide a framework that can guide future research and delineates prior research for scholars to progress from. For this purpose, we collected and reviewed the existent body of research in a structured manner. Afterwards, we developed the study's framework through a guided content analytical approach towards the collected literature. Both processes are further elaborated in the following.

\subsection{Paper Collection for Literature Review}

In conducting a scoping review (Paré et al. 2015) of blockchain research, we followed a systematic approach towards selecting and analyzing literature in this emerging research stream. Based on our research questions, we developed a protocol for identifying papers to be included in the analysis. In line with the idea of a scoping review, we thereby aimed for a comprehensive overview of prior work relevant to our research questions but willingly excluded even high quality papers on blockchain technology if they did not help answer our research questions (Paré et al. 2015). The protocol consisted of defined sources of research to scan, means to access them, and basic criteria for inclusion and exclusion of single papers (Kitchenham and Charters 2007; Paré et al. 2015). As we were interested in scientific knowledge on the wider ramifications of blockchain technology, we only focused on scholarly literature and thereby excluded the manifold statements, ideas, and visions of blockchain enthusiasts and opponents in public press, media, and whitepaper collections. In doing so, we acknowledge that there are enormously influential whitepapers that have shaped the discussion of blockchain in industry as well as academia (esp. Back et al. 2014; Buterin 2014a; Nakamoto 2008; Rosenfeld 2012; Schwartz et al. 2014; Wood 2014), but we refer readers to detailed extant discussions of these papers (Tschorsch and Scheuermann 2016; Yli-Huumo et al. 2016).

We searched the databases of the Web of Science, IEEE Xplore, the AIS Electronic Library, ScienceDirect, and SSRN for research on blockchain technology published in journals and conferences. In particular, we used the search terms "block chain" and "blockchain" in the mentioned databases. As there is a number of helpful syntheses on the state of technical research on blockchain protocols (e.g., Morisse 2015; Tschorsch and Scheuermann 2016; Yli- 
Huumo et al. 2016), we decided to focus only on papers that went beyond technical blockchain protocol improvements. We were particularly interested in finding conceptual papers or empirical analyses of the application, design, use, or implications of blockchain technology for humans, organizations, and markets. For reasons of quality assurance, we discarded all working papers and workshop proceedings to retain only published academic research in scholarly journal articles and conference proceedings. We then examined titles and abstracts of all retained papers for elements that referred to the application, design, use, or implications of blockchain technology for humans, organizations, or markets. All papers that matched any one of those criteria were included in our review. We explicitly excluded papers that solely focused on technology or on cryptocurrency performance or market trends. Technical papers improving or proposing algorithms without any connection to humans, organizations or markets were also discarded. In order to ensure consistency in the selection procedure, the first and second authors of this paper jointly defined the inclusion and exclusion criteria, examined a set of 10 papers together with a research assistant for their relevance, and then had the research assistant recommend selection or rejection for all identified papers based on their full text. The first and second authors then each examined half of the proposed selections and rejections based on their abstracts to verify the selection quality. In few cases of disagreement, the authors and the assistant discussed their opinions to come to a joint verdict about inclusion or exclusion (Paré et al. 2015). In order to address the critique of systematic literature reviews (Boell and Cecez-Kecmanovic 2014), we also reviewed the citations of selected papers to determine whether any of them referenced research papers that we had inadvertently overlooked in our initial selection process (Webster and Watson 2002). The authors lastly read the selected literature and removed papers that were not targeting the focus area as expected from the abstract. A qualitative content analysis was conducted for a final set of 69 papers. Figure 1 depicts the numbers of papers that emerged from the single steps of this process. In sum, we collected a broad set of literature from various disciplines that provided the input to our content analysis.

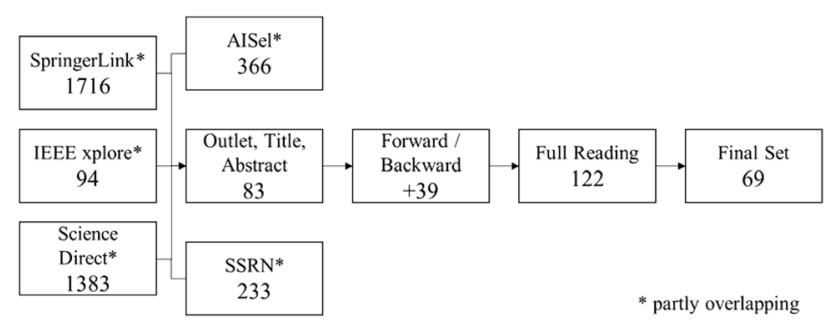

Fig. 1 Literature selection process

\subsection{Directed Content Analysis Approach}

In order to develop a framework for tracking and guiding blockchain related advancements, we oriented towards Morris' five-step process for directed content analysis as described below (Hsieh and Shannon 2005; Morris 1994). We based our approach on predefined categories and descriptions of a recognized research structure from the social media context (Aral et al. 2013), which enabled us to draw systematic and valid inferences from the collected data (Krippendorff 2012). This framework was selected, because it pursues corresponding purposes for a conceptually related technology. We consider social media technology to be conceptually related, because - comparable to blockchain - it provides a tool for transparent and largescale, many-to-many exchanges that empower the individual. In the case of social media, this complex and participatory environment has redirected control from companies to consumers and enhanced the users' ability to undertake collective action (Shirky 2011). Comparably, blockchain technology enables comprehensive bidirectional transactions, improves transparency, and is argued to pose disruptive challenges to society and central authorities (Atzori 2015). Thus, we assume the social media framework from Aral et al. (2013) to provide a viable starting point for structuring blockchain research.

Following Morris (1994) five steps, at first the singular studies were determined as the unit of analysis, which constitute the fragments in which the data was broken down to for the coding process (Rourke et al. 2001). We categorized them for their academic discipline and for their research method. Subsequently, we developed the categorization framework based on the established social media structure from Aral et al. (2013). Initially, the scheme was intensively discussed among two senior researchers familiar with the theoretical and technological background. Afterwards, it was applied to structure the collected studies, which led to some revisions under consideration of the blockchain specifics (Glaser 2017) alongside the test-classification process until the final version was created (further details can be found in Sects. 4.1 and 4.2). Lastly, the authors processed all 69 selected focal studies in accordance with the research framework.

\section{Results}

\subsection{Research Framework}

The framework (Table 1) is based on the popular social media research agenda from Aral et al. (2013). It was adapted to the blockchain context mainly by adjustments regarding the characteristic aspects and affordances of 
Table 1 Multidisciplinary blockchain research framework with prospective paradigmatic research questions

\begin{tabular}{|c|c|c|c|}
\hline \multirow{2}{*}{$\begin{array}{l}\text { Level of } \\
\text { analysis }\end{array}$} & \multicolumn{3}{|l|}{ Activities } \\
\hline & Design and features & Measurement and value & Management and Organization \\
\hline $\begin{array}{l}\text { Users and } \\
\text { society }\end{array}$ & $\begin{array}{l}\text { How do blockchain features and } \\
\text { design affect the interaction between } \\
\text { users and technology adoption? } \\
\text { How do different features constrain } \\
\text { or unchain usage? }\end{array}$ & $\begin{array}{l}\text { What are the benefits and costs of using } \\
\text { blockchain technology for the individual user and } \\
\text { the society? }\end{array}$ & $\begin{array}{l}\text { How to balance user privacy and } \\
\text { legal demands? } \\
\text { Why and how do users perceive } \\
\text { transactions with humans or } \\
\text { artifacts as sufficiently } \\
\text { trustworthy? }\end{array}$ \\
\hline Intermediaries & $\begin{array}{l}\text { How do alternative blockchain } \\
\text { features and designs enact different } \\
\text { intermediary services? } \\
\text { How do specific features } \\
\text { complement existing } \\
\text { intermediaries? }\end{array}$ & $\begin{array}{l}\text { How can blockchain systems maximize their role } \\
\text { as a transaction intermediary? } \\
\text { What are the value propositions and the } \\
\text { limitations of blockchain technology compared } \\
\text { to established intermediary services providers? }\end{array}$ & $\begin{array}{l}\text { How do existing intermediary } \\
\text { service providers position } \\
\text { themselves towards blockchain } \\
\text { technology? } \\
\text { Which business transactions can be } \\
\text { outsourced to blockchain } \\
\text { systems? }\end{array}$ \\
\hline Platforms & $\begin{array}{l}\text { How do blockchain platforms differ } \\
\text { regarding features and designs? } \\
\text { How can different blockchain } \\
\text { systems complement each other to } \\
\text { overcome individual constraints? }\end{array}$ & $\begin{array}{l}\text { How can blockchain systems enhance their } \\
\text { dissemination among users and linkage with } \\
\text { operating systems? } \\
\text { What are the complementary benefits of } \\
\text { blockchain systems to established information } \\
\text { systems? }\end{array}$ & $\begin{array}{l}\text { How can decentralized blockchains } \\
\text { establish and govern innovative } \\
\text { ecosystems? } \\
\text { What are the effects of hard forks? } \\
\text { How can they be managed or } \\
\text { prevented? }\end{array}$ \\
\hline $\begin{array}{l}\text { Firms and } \\
\text { industries }\end{array}$ & $\begin{array}{l}\text { How can firms utilize blockchain } \\
\text { features for their own business } \\
\text { processes? } \\
\text { What blockchain features are } \\
\text { relevant for different company } \\
\text { divisions or industry branches? } \\
\text { What type of blockchain is best- } \\
\text { suited for the respective purposes? }\end{array}$ & $\begin{array}{l}\text { How does blockchain provide added value for } \\
\text { companies to conduct transactions within the } \\
\text { company or with customers, other companies, } \\
\text { stakeholders and the government? } \\
\text { Which markets, industry branches, business } \\
\text { models or corporate divisions are more likely to } \\
\text { be affected by blockchain? }\end{array}$ & $\begin{array}{l}\text { How do organizations act under } \\
\text { different blockchain based regimens } \\
\text { of data privacy/confidentiality? } \\
\text { How does decentralized control } \\
\text { work in industry-wide blockchain } \\
\text { systems? } \\
\text { Can new forms of organization be } \\
\text { managed effectively on a } \\
\text { blockchain? If so, how and why? }\end{array}$ \\
\hline
\end{tabular}

blockchain technology (Glaser 2017). The framework is conceptualized as an intersection of activities that blockchain developers and users can undertake and the levels of analysis on which these activities wield influence. Relying on a powerful, established framework from related literature and transferring respective research questions enables us to systematically identify general research topics beyond the currently discussed research objectives. Thereby, we are able to raise themes that move beyond the current blockchain state-of-the-art and broaden the scope to topics that have not yet been considered by contemporary research reviews that solely focus on the subjects covered by the reviewed articles (Yli-Huumo et al. 2016).

The combinations of activities and levels of analysis in this framework provide analytical categories of technological and theoretical advancements regarding blockchain systems. It needs to be noted, that these categories are not defined to be mutually exclusive in the sense that a research objective can only address one activity and one unit of analysis at a time. These categories rather help to structure and inspire future advancements in blockchain research. They allow for the identification of neglected topic areas and the definition of meaningful implications for the respective analytical categories. Furthermore, due to the pervasive potential of the blockchain technology (Glaser 2017), it is expected to affect various different aspects of society (e.g., politics, business models). Therefore, we argue that it is beneficial for researchers to collaborate across disciplines or at least consider related theories and analytical contexts. Consequently, the developed framework also seeks to inspire cross-disciplinary research with contributions from separate fields of expertise (further elaborated in Sect. 4.3.1).

The framework's levels of analysis refer to the scale of the research target. In reference to Aral et al. (2013), we differentiate between the four perspectives: "users and society', 'intermediaries', 'platforms', and 'firms and industries'. As opposed to social media, blockchain systems are decentrally hosted providing a reliable infrastructure independent from the particular intermediary services provider (Glaser 2017). Thus, deviant from the original structure in the social media context, we consider intermediaries and platforms distinct from each other to account for this unique potential of blockchains as an 
intermediating technology (Yli-Huumo et al. 2016) and for the technological separability of fabric and application layers (Glaser 2017). Users and society refers to individuals who transact through blockchain applications and the societal consequences that the technology implies. Intermediaries refer to intermediary service providers as well as applications and processes that are hosted within a blockchain environment connecting a service provider and a service consumer. The key focus on this level revolves around smart contracts and the consequent opportunities for automating transactions among dispersed entities (Szabo 1997), for example, in the context of the Internet of Things or supply chain management. The category Platforms comprises different blockchain implementations and networks (e.g., Ripple, Ethereum, Hyperledger), various types of blockchains (e.g., permissioned vs. permissionless), as well as cross-system interactions (e.g., integrating blockchain systems with each other or into established systems). Lastly, Firms and Industries describe the organizations and industries that are prone to be affected by blockchain technology or deploy blockchain solutions themselves (e.g., financial markets, public services) as well as how (new) business models will develop in a blockchain industry. This level of analysis can be considered to account for the majority of the present hype around blockchain, as different industries try to assess the disruptive force that blockchain technology entails.

The activities regarding the Design and Features revolve around the questions of how blockchain systems are designed and what the differential effects of the various characteristics (e.g., consensus mechanisms, privacy settings, transparency, immutability, decentralized control) are. The overarching goal is to derive an understanding of how systems should be designed to achieve certain goals. Measurement and Value generally concerns the added value that blockchain based solutions provide on the different levels and how it can be appropriated. Work in this area assesses the benefits and competitive advantages that result from blockchain technology as well as how these systems challenge existing services and industries. Management and Organization addresses questions regarding the governance of decision rights in blockchain environments and the strategies and tactics employed by actors in blockchain systems. Specific topics in this area cover, for example, the development and implications of different consensus mechanisms, legal consequences accompanying transactions, organizational strategies, and patterns of participation in blockchain systems. In the original social media framework, strategy and tactics constituted a distinct activity. It referred to how the different entities use the technology to best achieve their goals (Aral et al. 2013). Our review of blockchain literature, however, revealed that respective endeavors are usually determined by the context.
Smart contracts, for example, are often characterized by the maxim "code is law". Strategic decisions on blockchain rather revolve around which blockchain, coin, smart contract to use for the individual purpose or how to best integrate it into operating services. These questions, however, are closely interrelated with the management and organization of the respective blockchain based system. Thus, while we acknowledge that tactical questions exist in the blockchain environment, we see them as interwoven with managerial and organizational decisions rather than as a distinct activity.

\subsection{Current State of Knowledge and Research Trends}

Blockchain technology is receiving a substantial amount of interest from researchers and practitioners. While research on some forms has rapidly developed (e.g., cryptocurrencies, payments), a comprehensive understanding regarding terms of application and use-cases is generally missing. Through reviewing extant findings and arranging them in accordance with the proposed framework, we structure the current knowledge and develop an agenda for future advancements in blockchain research [Table 2, Table A1 (in the appendix, available online via http://link.springer. com)]. Thereby, this work intends to calm the hype regarding societal and business implications while enabling the alignment of efforts - also across disciplines - to ensure impactful research.

\subsubsection{Design and Features}

The novel features that Blockchain introduces, for example regarding decentralized control and immutability of event $\operatorname{logs}$, determines the applicability and potential of the technology (Tschorsch and Scheuermann 2016). In our understanding, research on blockchain design and features forms the basis for the value and management propositions. It deals with identifying the unique blockchain features and explicating their respective impacts. As our analysis shows, this area is currently the most heavily investigated research stream helping to understand the technological basics. Drawing on the blockchain archetype framework by Walsh et al. (2016), we review the respective literature regarding the features that distinguish different blockchains (i.e., consensus mechanisms, types of permissioning, data access, modularity, scalability, interoperability, centralization, and anonymity).

Users and society Questions regarding this topic address how users perceive and interact with different blockchain characteristics. As a key topic, research needs to provide insights on why people use the technology and what features enhance or constrain its dissemination among the society. In their particular context, for example, system 
Table 2 Results of the blockchain research classification based on the research framework

\begin{tabular}{|c|c|c|c|}
\hline \multirow[t]{2}{*}{ Level of analysis } & \multicolumn{3}{|l|}{ Activities } \\
\hline & Design and features & Measurement and value & Management and organization \\
\hline Users and society & $\begin{array}{l}\text { Abramova and Böhme (2016) } \\
\text { Fabian et al. (2016) } \\
\text { Yli-Huumo et al. (2016) } \\
\text { Walch (2017) }\end{array}$ & $\begin{array}{l}\text { Beck et al. (2016) } \\
\text { Nguyen (2016) } \\
\text { Pilkington et al. (2017) }\end{array}$ & $\begin{array}{l}\text { De Filippi (2016) } \\
\text { Kiviat (2015) } \\
\text { Maesa et al. (2016) }\end{array}$ \\
\hline Intermediaries & $\begin{array}{l}\text { Gipp et al. (2016) } \\
\text { Hashemi et al. (2016) } \\
\text { Juels et al. (2016) } \\
\text { Kosba et al. (2015) } \\
\text { Mainelli and Smith (2015) } \\
\text { Watanabe et al. (2015) } \\
\text { Yasin and Liu (2016) } \\
\text { Zhang et al. (2016) }\end{array}$ & $\begin{array}{l}\text { Korpela et al. (2017) } \\
\text { Feng (2016) } \\
\text { Zhang and Wen (2015) }\end{array}$ & $\begin{array}{l}\text { Fujimura et al. (2015) } \\
\text { Lewenberg et al. (2015) } \\
\text { Raskin (2016) } \\
\text { Reyes (2017) }\end{array}$ \\
\hline Platforms & $\begin{array}{l}\text { Danezis and Meiklejohn (2016) } \\
\text { Gervais et al. (2016) } \\
\text { Glaser and Bezzenberger (2015) } \\
\text { Kazan et al. (2014) } \\
\text { Tschorsch and Scheuermann (2016) } \\
\text { Walsh et al. (2016) } \\
\text { Watanabe et al. (2016) } \\
\text { Xu et al. (2017) } \\
\text { Zhu et al. (2016) }\end{array}$ & $\begin{array}{l}\text { Hayes (2016) } \\
\text { Lindman et al. (2017) } \\
\text { Sanda and Inaba (2016) } \\
\text { Xu et al. (2016) }\end{array}$ & $\begin{array}{l}\text { Cocco and Marchesi (2016) } \\
\text { Decker and Wattenhofer (2013) } \\
\text { Dennis and Owen (2015) } \\
\text { Dwyer (2015) } \\
\text { Reyes (2016) } \\
\text { Rückeshäuser (2017) } \\
\text { Zou et al. (2016) }\end{array}$ \\
\hline Firms and industries & $\begin{array}{l}\text { Aitzhan and Svetinovic (2016) } \\
\text { Brandon (2016) } \\
\text { Glaser (2017) } \\
\text { Mettler (2016) } \\
\text { Morisse (2015) } \\
\text { Wörner et al. (2016) }\end{array}$ & $\begin{array}{l}\text { Ainsworth and Shact (2016) } \\
\text { Azaria et al. (2016) } \\
\text { Brenig et al. (2016) } \\
\text { Christidis and Devetsikiotis (2016) } \\
\text { Morini (2016) } \\
\text { Nofer et al. (2017) } \\
\text { Lee and Pilkington (2017) } \\
\text { Sikorski et al. (2017) } \\
\text { Yermack (2017) } \\
\text { Yuan and Wang (2016) }\end{array}$ & $\begin{array}{l}\text { Beck and Müller-Bloch (2017) } \\
\text { Bell (2016) } \\
\text { Caytas (2016) } \\
\text { Lee (2016) } \\
\text { McJohn and McJohn (2016) } \\
\text { Paech (2016) } \\
\text { Peters et al. (2015) } \\
\text { Shackelford and Myers (2016) } \\
\text { Vogel (2015) }\end{array}$ \\
\hline
\end{tabular}

providers are interested in the relative importance of different features (e.g., privacy, security, usability, latency) that determine end-user adoption. Regarding anonymity, deanonymization attacks through analyzing transaction logs (Meiklejohn et al. 2013; Ron and Shamir 2013) are argued to be a major technology adoption hindrance (Kosba et al. 2015). Privacy and security related issues could, for example, also be moderated by cultural (King and Raja 2012) or age related differences (Hoofnagle et al. 2010). Furthermore, research may need to critically examine the uncritically accepted assumption that people generally appreciate the trust-free characteristics found on different blockchains. Importantly, it is not even clear whether blockchain transactions are actually perceived as trust-free since they may still require a certain amount of trust into the blockchain providers or smart contract developers (Glaser 2017).

Regarding the level of anonymity (Walsh et al. 2016), Fabian et al. (2016) found in a recent survey that anonymity serves as a double-edged sword in blockchain based transaction. For example, while the majority of active Bitcoin users report minor concerns with the network's anonymity, almost $20 \%$ consider abandoning the technology because of it. Future research will need to investigate how this adverse effect can be mitigated and where it stems from. Further research interests address blockchain scalability. After developing a blockchain dependent solution for coffee-shop payments, Beck et al. (2016) argue that scalability issues, costs, and volatility in the transaction currency can constrain the adoption and utilization. Other 
researchers have shown that scalability issues are closely related to security issues and that trade-offs may be necessary between these two dimensions depending on the consensus mechanisms that single blockchains entail (Anceaume et al. 2016). Therefore, Buterin (2014b) proposed a system of multiple, different blockchains that provide security for each other irrespective of their distinct purposes. This would help overcome security issues that limit the scalability of singular blockchains for society. Regarding the effects of decentralization, Abramova and Böhme (2016) found that decentralization constitutes the smallest perceived technological benefit among Bitcoin users compared with faster transaction processing and control over money. Thus, it can be concluded that first research has begun to identify features that support and restrain the blockchain appropriation. The revision of these different technological blockchain features and issues points towards currently discussed (e.g., Decker and Wattenhofer 2013) and scientifically further investigated technological issues [e.g., latency, throughput, blockchain versioning (Yli-Huumo et al. 2016)]. Applying the blockchain archetypes framework (Walsh et al. 2016) shows that while anonymity, decentralization and scalability have initially been investigated, other aspects such as effects of (un-) permissioned blockchains, restricted data access, consensus mechanisms, modularity, and interoperability are mostly disregarded. Furthermore, the currently insufficient technological understanding translates into legislative risks (Walch 2017), which substantially affects the individual adoption of blockchain technology (Abramova and Böhme 2016). Therefore, future research needs to acknowledge the distinct features of different blockchains (e.g. consensus mechanisms, block sizes, permissioning) to understand their respective application consequences (e.g. for scalability, security, privacy). More comprehensive research is needed to fully understand the underlying mechanisms and to be able to identify means of overcoming these obstacles in order to advance the technology's dissemination.

Intermediaries Blockchain technology offers swift implementations of automated transaction management with comparatively little coding effort. In this area, the blockchain application layer that provides intermediary services is of primary interest. The focus lies on the design of smart contracts and the development of decentralized applications (DApps) that run on them (Glaser 2017). Respective studies can, for example, identify or design different application features (e.g., permission requirements) or integrate blockchain based solutions into established systems (e.g., enterprise resource planning, account management systems) and evaluate their performance (e.g., regarding operational reliability).
Design related blockchain research on the intermediary level generally investigates the intersection of interoperability and anonymity. In an explorative approach, Mainelli and Smith (2015) concluded that integrating distributed ledgers into trusted third party systems can support services such as know-your-customer, money-laundering prevention, insurance or credit services. Scientists develop easily implementable protocols for smart contracts that pool transactions in order to anonymize single transactions and protect individual privacy (Kosba et al. 2015), manage healthcare (Zhang et al. 2016) and IoT applications (Hashemi et al. 2016) or enable contract recording (Fujimura et al. 2015) and tamper-proof dashboard video transmission (Gipp et al. 2016). Unfortunately, however, anonymously running smart contracts can also be applied for criminal purposes (e.g., information leakage, private key theft, real-world crimes) difficult to prevent through countermeasures (Juels et al. 2016).

While first studies show the relevant and productive opportunities of these aspects, a lot more research will be necessary to properly address these practically relevant topics. Blockchain features such as levels of permission, data access, consensus mechanisms, scalability, and decentralization (Walsh et al. 2016) are generally neglected in this regard. With a growing number of available applications, we expect this field to gain momentum. So far, it seems that the developed blockchain based solutions rather provide new systems instead of replacing or complementing existing ones. We encourage research in the future to consider the compatibility of services.

Platforms This line of work focuses on classifying and advancing the different technological mechanisms underlying different types of blockchains, platforms, and networks on the fabric layer that is also addressed in technical whitepapers (esp. Back et al. 2014; Buterin 2014a; Nakamoto 2008; Rosenfeld 2012; Schwartz et al. 2014; Wood 2014). Features of interest comprise consensus mechanisms, permissioning of writing or reading rights, scalability mechanisms, decentralization, levels of anonymity and interoperability. Thereby, different interdependencies between features need to be considered. For example, private blockchains can make use of more lightweight consensus mechanisms than public blockchains by relying on a certain level of trust in participants (Buterin 2015). This allows them to rebalance efforts for security with efforts for speed and scalability, for example. So far, however, research has not yet provided a systematic overview regarding these interdependencies and their consequences for use cases. Different blockchains implement various consensus mechanisms for validators. Research needs to identify the impact of these consensus mechanisms for the appropriation in different business cases and the (dis-)advantages of open-source vs. 
proprietary blockchains. Furthermore, research will need to identify means of integrating blockchain platforms into established systems (e.g., aligning a real world dividend payment system with blockchain based token distribution; data transmission between corporate internal auditing systems with governmental taxation systems) or integrating complementary blockchain systems (e.g., integrating Hyperledger Fabric with Ethereum). Regarding the constituting features, future work can critically revise the optimal block size or the respective cryptographic security measures depending on the specific context of implementation. This could lead to an advancement of the hashing algorithms to prepare the different platforms to increasing security challenges from, for example, distributed denial of service attacks (Coleman 2016). Lastly, we expect to have a discussion in this area on what actually constitutes a blockchain. First authors have already critically noted that the recently developed (permissioned) blockchains do not constitute blockchain systems in the original sense (Glaser 2017). This controversy will increase as further blockchain systems (e.g., the IBM blockchain system operated in a hosted cloud environment) enter the market.

First approaches introduced blockchain technology to research by providing overviews over (de)centralized consensus systems in the context of cryptocurrencies (Glaser and Bezzenberger 2015; Tschorsch and Scheuermann 2016), blockchain typologies (Walsh et al. 2016; Xu et al. 2017) or digital payment providers in general (Kazan et al. 2014). These provide detailed overviews over the differentiating characteristics of alternate platforms or elaborate their cryptographic foundations to analyze the applicability to policymaker regulations (Kiviat 2015). Focusing on particular blockchain properties, some studies have advanced insights on consensus mechanisms. Generally, it has been established that the proof-of-work based consensus mechanism applied by Bitcoin developers - as a prominent blockchain application example - sacrifices a substantial amount of transaction speed and volume for little incremental security (Gervais et al. 2016). By scripting a signature that assures transaction non-repudiation (Zhu et al. 2016) or proposing a consensus mechanism for contract management that is robust against resource monopolization (Watanabe et al. 2016) researchers were able to increase transaction security. Others focus on the scalability related feature of hashing to overcome the security issue of double spending. Danezis and Meiklejohn (2016) discuss different forms of centralization within a cryptocurrency framework to show that the reduction of inefficient hashing and a scalable system due to a modest degree of centralization can reduce the danger of double spending attacks. Others focus on the cryptographic properties by developing solutions to stabilize block rates over longer periods of time by manipulating hashing difficulties (Kraft 2016).

Research on platform design and features has successfully contributed to the public understanding of the applicability of blockchain technology and its characteristics. Most prominently, research has investigated approaches to increase transaction security and consensus mechanisms, scalability, and partly decentralization. Other blockchain features such as levels of permissioning, data access, modularity, interoperability and anonymity (Walsh et al. 2016) are frequently researched in industry but have received less academic attention. Considering the rapid developments, this topic area can be expected to continuously evolve and advance. Particularly the maturation of cryptographic foundations, for example, towards a proofof-stake (Back 2017) and assessing the consequences for the scalable applicability of blockchain technology is of ever-growing interest.

Firms and industries The features of blockchain technology are considered to make it potentially disruptive for many different businesses processes and industries. However, little is yet known regarding which (combinations of) features are relevant for particular industries and how they need to be designed. These types of questions need to be addressed in order to influentially deploy the technology to business cases. A blockchain system in the context of Scottish stock-trading settlement (Detrixhe 2016), for example, will have block size and confirmation speed requirements that differ from those for settling public services (Finley 2016). These findings will inform and inspire further business related research regarding (dis)advantages and possibilities of home-grown and externally hosted blockchains. Beyond effects on established businesses and services, blockchain also enables new business models like decentralized autonomous organizations (DAOs) or decentralized autonomous corporations (DACs). Their prospects also depend on the underlying computational design (e.g., security features), as was recently shown by a substantial capital loss due to flawed system design (Price 2016).

Research in this area principally revolves around the general blockchain features. Glaser (2017), for example, argues that immutability will provide major benefits for auditing services, where only a permissioned blockchain that reduces transparency constitutes a feasible solution. This claim was extended further to the accounting discipline (Brandon 2016). Other work has begun to discuss the disruptive potential of general blockchain features (i.e. immutable public database, time-stamping service, verifiable audit trails, decentralized infrastructure) for different sectors like digital assets, marketplaces, and notary services (Korpela et al. 2017; Wörner et al. 2016), the energy (Aitzhan and Svetinovic 2016) or healthcare sectors 
(Mettler 2016). In the context of cryptocurrencies, these findings can be expanded in accordance with the technological classifications derived from the overviews of cryptocurrencies (Glaser and Bezzenberger 2015; Morisse 2015). Only recently it was pointed out that the discussion of business applicability needs to consider different blockchain features as, for example, not all consensus mechanisms match industry-specific requirements (Rückeshäuser 2017).

It can be concluded that previous work on firms and industries has provided extensive frameworks and starting points for future research to advance research in a structured and impactful fashion. However, a more sophisticated consideration of the differentiating blockchain features - namely the level of permission, data access, transaction consensus, modularity, scalability, interoperability, centralization, and anonymity (Walsh et al. 2016) is needed. Moreover, research on the interaction of new organizational forms such as DAOs and technological features of blockchain are still to come. This stream of research has the potential to provide meaningful guidance for the design of increasingly versatile solutions that enter the market.

In general, the overarching analysis of research on blockchain design and features shows that tremendous effort has produced first insights into the particularities of blockchain technology. Particularly centralization, anonymity, consensus mechanisms and scalability have been predominantly investigated, while other features like data access, modularity, and interoperability have received less attention. These approaches, however, seem to be rather incoherent. For example, different levels of anonymity and the perception of anonymity are typically only investigated on the user level. Interoperability has only been researched on the intermediary level. Only scalability issues have been discussed on most units of analysis. We argue that a structured and comprehensive overview of interdependencies of blockchain design and features will - as a first step help to provide a solid foundation for future research and as a second step - to systematically discuss the relation between blockchain features and design on the different levels of analysis.

\subsubsection{Measurement and Value}

This line of research generally addresses the added value that blockchain produces for users and industries under consideration of platforms and applications. While aforementioned literature has provided first insights regarding the blockchain design, economic consequences are usually assumed but not demonstrated. Regarding the value related to blockchain technology, most of the discussion has revolved around cryptocurrencies, especially Bitcoin.
However, identifying the unique value that blockchain technology provides compared to established systems is arguably among the most intensely conversed topics in this area. In this regard, research will also need to investigate the value and cost of integrating blockchain based solutions into existing information systems, considering that the switching costs might deter people and organizations from migrating entire systems or services onto blockchain systems (Shin 2016). Beyond the benefits of blockchain technology, research will also need to weigh the expense at which the respective surplus comes. Gaining transparency, for example, could also demand a trade-off with reduced anonymity due to higher identifiability through transaction pattern recognition or user meta-information. These deliberations ultimately lead to the question of value measurement. While the economic return on investment is the most commonly demanded measure by practitioners, researchers should also investigate the relative importance of and impacts on other ascertainable metrics (e.g., ease of use, trustworthiness). Considering blockchain technology's core functionality of providing validated and immutable transactions, projects in this area should generally first identify which types of transactions could benefit from blockchain affordances and then assess how these improvements can be measured. Currently, however, literature provides only few convincing use cases (Glaser 2017).

Users and society Digitalization is expected to be the major disruptive force for modern society in the years to come. Due to the advantageous efficiency of programmable processes, digitalization is believed to reshape even knowledge-intensive industries and services (Loebbecke and Picot 2015). Due to its potential pervasiveness (Glaser 2017), especially blockchain technology represents a potent driver of this development. Researchers need to investigate the associated costs of blockchain systems for individuals and the society (e.g., reduced anonymity, loss of jobs) to enable, for example, policy makers take reasonable measures addressing these risks. Beyond potential costs, blockchain also offers ascertainable benefits for the individual. It is argued that this added value comprises the facilitation of payment services (Beck et al. 2016), profound knowledge on product background (Finley 2016), and inexpensive intermediary services (Tapscott and Tapscott 2016). Thus, it can be seen that the potential risks and benefits of blockchain are manifold. Identifying and measuring the potential value of blockchain systems for the individual will also be of interest for businesses, which need to decide how this technology can help them provide more efficient and leaner services to their customers.

Beck et al. (2016) were able to provide a first proof of concept for a blockchain system that facilitates the payment process for customers in the case of a coffee shop. At 
the same time, however, they also showed that individual level adoption hindrances are a key determinant for the system success or failure. Apart from this first use-case implementation, researchers generally focus on conceptualizing the potential societal benefits of blockchain to diminish political corruption (Pilkington et al. 2017) or revolutionize the banking sector to enable a sustainable global economy (Nguyen 2016). Beyond the first practical approach, however, little has been demonstrated regarding the individual level costs and benefits of blockchain technology. Existent publications rather focus on to the conceptualization of potential societal benefits of blockchain technology. More practical implementations and considerate empirical investigations are needed to substantiate these claims.

Intermediaries As mentioned earlier, a core affordance of blockchain technology is its potential to improve intermediated transactions in general. Smart contracts enable autonomous mediation between transaction partners without the need for trust into the other party. Thereby, blockchain technology is argued to provide inexpensive alternatives to classical intermediary services providers [e.g., credit card companies, stock exchanges (Glaser 2017)]. This introduces a broad range of questions, for example on how blockchain applications can replace intermediary services providers or whether the established companies can implement blockchain-based solutions to complement their current business. Regarding procurement, for example, features such as transparency and immutability enable the unique value propositions of the blockchain based intermediary service provider Everledger (Price 2015). By identifying the value propositions and the limitations that blockchain technology offers compared to established intermediary services providers, research can also shed light on which business models are actually going to be challenged by blockchain-based systems (e.g., notary, financial industry). Furthermore, other current digitalization advancements can benefit from blockchain. Thus, not only established businesses can be changed but also new business opportunities may arise through blockchain based applications. The most prominent case are current developments regarding the Internet of Things where blockchain is argued to introduce a new platform technology that provides the missing link for privacy, reliability and scalability for the rising technological trend (Banafa 2016).

In a first approach, Christidis and Devetsikiotis (2016) actually indicated the potential of smart contracts to safely support transactions between devices in the context of the Internet of Things. They are able to derive temporal advantages through cryptographically verified automated system interactions. But also in real-world settings, blockchain technology transaction processing and timestamping has been found useful for supply chain management in general (Korpela et al. 2017) and in combination with RFID technology for the food chain in particular (Feng 2016).

Beyond these findings, however, no studies have actually investigated questions of blockchain's value for intermediary service provisioning. As the technology development progresses and business cases arise, we expect this to be of major interest for academic and practitioner communities. Particularly the combination with transmitter technologies (e.g., RFID, beacons) constitutes a great potential for supply chain management automation. Thus, researchers should consider implementing respective applications and empirically measuring value created in real life settings.

Platforms After different types of systems have been established, it will be necessary to determine the unique surplus these systems provide. For example, considering parallels to physical currencies where political borders influence the scope of value, little is yet known regarding the convertibility of cryptocurrencies or even other digital assets across platforms. Seeing that different platforms implement their unique tokens that correspond to different valuta, researchers need to inform the process of managing value between multiple currencies. These findings will also help monetary authorities in developing proper means of integrating cryptocurrencies into established systems and regulating cryptocurrency exchanges. Furthermore, drawing the comparison between blockchain networks and social media platforms, it will be necessary to investigate the differences between environments. Thereby, their respective added value can be identified in order to enable users and companies make educated decisions on which platform to engage for attaining their respective goals. In this context, it will also be necessary to determine their complementary values in order to be able to judge the sustainability of these systems. For example, the integration of an Instagram account into one's Facebook profile is rather inconsequential compared to a migration of a potentially affluent depot from one Ethereum-based blockchain system to another in the case of a blockchain merger. A deepened understanding of these networks for the public and businesses could then again increase their willingness to engage even on public platforms.

First scientific approaches in this particular regard have focused on outlining research questions towards understanding the blockchain potential as a digital payment system (Lindman et al. 2017) up to discussing its capability of replacing a central bank (Hayes 2016). On a lower scale focus, researchers have proposed a framework towards analyzing the integration of blockchain-platforms into existing software solutions (Xu et al. 2016) or actually integrated a blockchain system to safely encrypt open WiFi hotspots (Sanda and Inaba 2016). 
While these approaches outline the added value of blockchain technology for different industries, barely any research has practically addressed the issue of integrating blockchain-platforms into operational information systems to complement and improve services. The measurement of generated value has largely been confined to cryptocurrency market trends on their respective platforms.

Firms and industries Insights regarding the impact of blockchain technology on business values are probably of the highest public interest at the moment. Questions in this area predominantly address which markets or industries will be affected by blockchain systems and how business models need to be designed to derive economic value from blockchains. While the financial markets is most commonly discussed (Holotiuk et al. 2017; Vernon 2016; Yermack 2017), other areas like logistics (Allison 2016) or public services (Ølnes 2016) move into focus as decision makers realize the potential for added value from this technology. The upcoming business models can be differentiated into enabling transactions between companies horizontally across the supply chain (e.g., R3 in the financial market) or within the companies' value chain (Science 2016). Currently, however, these assumptions are still only idea driven, and first skeptical corporations are withdrawing their investments from such business models (McLannahan 2016).

First research groups have taken on the substantial questions of blockchain value for firms and industries and are engaging in empirical research beyond mere conceptual discussions. As such, Beck and Müller-Bloch (2017) interviewed high ranking decision makers from large corporations to systematically develop a process of how blockchain technology can successfully be introduced within companies to generate business value. Brenig et al. (2016) develop a framework for the assessment of the business models of decentralized consensus system operations and Glaser (2017) provides a structure to systematically assess blockchain use cases. Similarly, others have started to understand industry-specific affordances of blockchain through structured data collections (Holotiuk et al. 2017; Korpela et al. 2017). In combination with IoT services, blockchain is esteemed to have substantial transformative power across several industries (Christidis and Devetsikiotis 2016). Beyond these conceptual approaches, researchers have investigated the blockchain business value in various different industries such as transportation (Lee and Pilkington 2017; Yuan and Wang 2016), financial industry (Morini 2016), electricity market (Sikorski et al. 2017) or the e-health sector (Azaria et al. 2016). But also public services can benefit from blockchain applications, for example in the case of EU-wide tax evasion-proof VAT collection (Ainsworth and Shact 2016).
These first studies provide increasingly reliable insights into the currently most discussed topic regarding the business value of blockchain technology and how to leverage its disruptive force. Building upon these studies provides a promising avenue for high impact studies necessary to substantially advance blockchain research. In particular, extensive empirical studies would be desirable to move the discussion of the value of blockchain technology to firm grounds.

\subsubsection{Management and Organization}

This line of research is concerned with questions surrounding the governance, use, effects and overall organization of blockchain based information systems. As such, it includes research that aims to understand the strategies and tactics employed by actors working on a blockchain as well as research that develops policies for integrating blockchain technology into current and future economic and societal settings. We expect that questions in this realm will also arouse the interest of multidisciplinary teams of researchers and will benefit particularly from collaborations of IS researchers with scholars from organization, management, political sciences, and law.

Users and society Decentralized networks of cryptography-based economic activity are a relatively new phenomenon, and societies need to understand the potential liberties and restrictions that come with them. General public and policy makers have recently been showing interest in cryptocurrencies and their interfaces to national currencies and electronic markets. In particular, legislative institutions around the world are trying to devise measures that prevent money laundering, fiscal fraud, and illegal activities in darknet marketplaces (Kiviat 2015). Societies and national states thereby try to apply their established systems of legal rules unchanged to blockchain systems that are largely based on pseudonymity of users and network-wide transactions irrespective of physical locations. The discussion to which degree such a transfer of rules is possible, and actually desirable, is so broad and impactful that we believe researchers from IS should engage with scholars of law and political sciences to bring together expertise in socio-technical, political, and legal matters necessary to drive this discussion in a competent way. Against this backdrop, the analysis of Kiviat (2015) brings to attention that currently devised regulatory approaches for cryptocurrencies have the potential to restrict the general applicability of blockchain technology for its even more powerful purpose: the exchange of digital assets. In order to prevent such collateral effects of legislation, scholars who are knowledgeable in socio-technical basics of blockchain technology should join the discourse and analyze proposed measures (Kiviat 2015). Given our 
discipline's focus and history, we see IS researchers well equipped to do so.

Beyond cryptocurrencies, blockchain based solutions have recently been discussed as a means for decentralizing political power and enabling truly democratic participation. Pilkington et al. (2017), for example, provide detailed concepts and evaluations how existing and emerging blockchain based projects may aid in fighting the effects of corruption and in increasing the social welfare in the Republic of Moldova as an example of a developing country. They point out that corruption particularly thrives when information is opaque and easily manipulated. To alleviate such antecedents of corruption, blockchain based systems need to be open and freely auditable, rather than permissioned and verifiable by few like some proposed closed systems (Pilkington et al. 2017). At the same time, there are also critical voices that do accept blockchain technology's potential for affecting even our current conception of national states but call for careful evaluations whether decentralized decision making indeed leads to more power for the individual or in fact to more privatized monopolies and a loss of common good and collective rights (Atzori 2015). This suggests a tremendous need for research that helps clearly identify and understand the strategic decisions that governments or even societies need to make when conceptualizing and introducing blockchain based services. First scholars of law are engaging in these emergent discussions both from normative and from analytical points of view (e.g., Raskin 2016; Reyes 2017; Savelyev 2017).

This discourse is closely related to the rapidly growing technical research stream that focuses on increasing data privacy on blockchains (cf. Yli-Huumo et al. (2016)). Although single users currently act pseudonymously based on their unique cryptographic keys in most blockchain implementations, the distributed and replicated nature of blockchain technology by and large exposes transactional data and the contents of smart contracts to all nodes of the network. Data analytics can therefore be used to gain insights into activities of single users as well as entire blockchain systems. While this can be seen as a strength regarding auditability, it can also be viewed critically from a privacy perspective (De Filippi 2016). Prior work on the network of users on the Bitcoin blockchain has, for example, shown that prominent nodes in blockchain transactions can often be identified as specific persons or organizations (Maesa et al. 2016; Yli-Huumo et al. 2016). The question where more or less anonymity of users is not only technically feasible but also desirable from an individual or societal perspective requires research to understand the behavior of networked individuals and groups under different levels of anonymity. Streams of IS research that have previously helped understand how even perceived anonymity fosters deviant behaviors of social media users (Lowry et al. 2016) may be fruitfully used and extended in this context.

Although well established IS methods and tools may be used to analyze blockchains in which human actors interact with other individuals, organizations, as well as technological artifacts like smart contracts, only few researchers have done so. For instance, Maesa et al. (2016) conduct a social network analysis of the Bitcoin blockchain. Their results suggest that characteristic deviances in the social network structure of Bitcoin from the social network structure of Facebook and other established social networks could be rooted in attempts to conceal real asset transactions between users. Scrutinizing and refining these results may therefore be of great interest to regulators and fiscal authorities. Finding ways to balance legal authorities' rights of inspection of asset transactions and the individual blockchain user's data privacy is therefore not only a technical question that should be worked on by software engineers and computer scientists. It is a socio-technical question that should also encompass studies of users and social networks who interact and conduct economic transaction based on specific blockchain technology.

Finally, blockchain implementations may actually provide the chance to study some uncharted areas of user behavior and human computer interaction. As such, blockchains will provide a platform even for complex business transactions between individuals and fully or partly autonomous technological agents like DAOs. Even today, human actors can invest in blockchain based programs that autonomously manage physical art objects, their monetization, and even their evolutionary development (Lotti 2016). It will be interesting to examine why and how individuals determine transactions with such technological actors as trustworthy. On the one hand, explicit and readably coded smart contracts may reduce uncertainty and the need to trust transaction partners, taking for granted that the transactions can only take place in the programmed way. On the other hand, the lack of legal enforcement possibilities should increase uncertainty and make individuals search for trustworthy transaction partners. Given the IS discipline's long history in researching trust in technology-mediated settings, we expect IS to make significant contributions in understanding how humans come to trust such new forms of organizations and their offers.

Intermediaries Although intermediary service providers are most likely the organizations whose business models will first be disrupted by blockchain based, automated solutions (Glaser 2017), there is comparatively little research on the strategies and tactics that intermediaries apply to benefit from blockchain technology or, at least, lessen the damage it causes to their business. This dearth of research may partly be rooted in a lack of existing and 
observable productive blockchain solutions by traditional intermediary service providers. Importantly, valuable solutions involve not only strong internal changes at intermediaries but also new intra- and inter-organizational collaborations (Beck and Müller-Bloch 2017). Solutions currently under development may therefore take some time to become productive. Nonetheless, scholars are expecting strong changes to the current state of intermediaries and accordingly also to the needs for legal boundaries of intermediaries (Vogel 2015).

Conceptual analyses suggest that the services of some intermediaries in multisided markets could be fully provided by relatively complex smart contracts (Glaser 2017). Even if these intermediaries were the ones to develop those smart contracts, it would be hard for them to charge their traditional amount of service fees, as they would always need to fear a less costly community solution. One elegant way to deal with this problem may be inherent to many blockchain implementations already: cryptocurrency-based reward mechanisms. Intermediaries may possibly use them to bind customers and service providers to their platform in order to generate network effects while simultaneously reducing operating costs by outsourcing blockchain operations to them. This approach has been demonstrated in prototypical implementations and few startups. For example, Yuan and Wang (2016) describe a case where validation of the blockchain transactions is provided by the so incentivized service providers in a ride-sharing network (i.e., by the drivers). Azaria et al. (2016) describe a prototype where data providers incentivize healthcare institutions and researchers to run and validate an infrastructure for exchanging encrypted patient data by providing them with anonymized, aggregated patient data as a bounty. Lastly, intermediaries could use the smart contract structure of DAOs to make their complementors shareholders of the intermediary. Doing so, they could however create even more open legal questions related to blockchain technology, particularly regarding the questions of who is liable for service provisioning and in case of fraud. We suggest that these questions be addressed in close collaboration with scholars of law.

Despite valuable first insights (Glaser 2017), it is currently largely unclear for which intermediaries public or private blockchain systems constitute a threat or opportunity. Based on the affordances and constraints of blockchain technology, research should consequently continue investigating which services provided by intermediaries can reasonably be programmed and automated if behavioral uncertainty of the parties is reduced and which services become obsolete if data can be shared directly through distributed, tamper-proof event databases. In fact, this could be a very valuable application for theory-guided action research. Given our discipline's theoretical, methodological, and practical expertise in IS outsourcing, we deem this also one very important area for empirical IS research to make valuable contributions.

Platforms Single implementations of blockchain technology differ amongst other things in their openness regarding their permissioning systems, their interfaces to external systems, as well as their incentive and consensus mechanisms (Tschorsch and Scheuermann 2016). In their entirety, such technological choices enable and constrain different behaviors of actors on these blockchains. On the public Bitcoin blockchain, for example, technological choices have so far stimulated a tendency towards conglomeration of mining activities (Tschorsch and Scheuermann 2016) that may eventually endanger the decentralized control of the network through monopolization. Simulation models that can be used to analyze or even predict such phenomena have, however, grown quite complex and specific. For example, Cocco and Marchesi (2016) succeed in reproducing many developments on the Bitcoin blockchain. In order to do so, however, they need to simulate not only specifics of the Bitcoin protocol but also technological advances in the hardware used for Bitcoin mining, archetypical behaviors of Bitcoin traders, and a price formation mechanism for Bitcoin (Cocco and Marchesi 2016). Although game theory traditionally provides a dependable foundation for analyzing consensus mechanisms in blockchain technology (Tschorsch and Scheuermann 2016), it is questionable how easily results from such fitted simulations can be generalized across single implementations of blockchain technology. IS research should therefore complement extant approaches to studying single blockchain platforms by bringing in theories and methods that have successfully yielded generalizable results in similar research streams such as on social media platforms and software ecosystems.

Particularly findings from software ecosystems literature (Agarwal and Tiwana 2015) may be helpful to understand how to organize and manage blockchain systems. In turn, platforms based on blockchain technology may be an interesting area of research for scholars of platform ecosystems as they clearly share several characteristics with traditional software ecosystems (e.g., the ones managed by SAP, Oracle, Apple, or Google) but also have distinctive properties: similar to traditional ecosystems, public blockchains need to attract and retain complementors to spur innovation and provide value-added services on top of the platform infrastructure in order to attract actually paying users. Similarly, public blockchains differ in their specific degrees of openness and modularity, in their technology-enforced rules what complementing smart contracts can do and what not. Contrastingly, however, public blockchains are distributed systems and do not have a single owner that can freely decide on changes to the 
platform or easily exclude insubordinate complementors. In contrast to traditional platforms, blockchain systems have a specific state and history of transactions, which are very hard to tamper with. These differences to traditional platforms become obvious when running blockchains are to receive major updates or when historical transactions are to be changed, for example after a successful hacker attack. For blockchain systems, such maintenance procedures, comparatively trivial in centralized systems, need the consent and active acceptance of all validating nodes in order to be effective. Without such consent, these procedures can actually result in a split of the chain so that two versions with competing transaction histories stay active until abandoned by all validating nodes (i.e., a hard fork, see Tschorsch and Scheuermann (2016)). On the one hand, open source research suggests that forks can have negative motivational consequences for developers in a project (Stewart and Gosain 2006) which could also apply to complementors on a blockchain. On the other hand, hard forks could signal that a public blockchain is able to react even to seemingly catastrophic events, making it more attractive for complementors. In case of hard forks that result in two enduring blockchains, some users may moreover be able to spend their historically accumulated digital assets twice, once on each version of the blockchain. There is little research on what such hard forks do to existing blockchain systems, their users, their complementors, and even the virtual organizations residing on them (Decker and Wattenhofer 2013). IS researchers should use their expertise on software ecosystems to address such important behavioral questions. Scholars targeting this fruitful area of research can find further valuable guidance in a research agenda on blockchains as platforms provided by Lindman et al. (2017).

Firms and industries Although many contemporary technical proposals of blockchain systems from research target specific industries (e.g., Yuan and Wang 2016; Zhang et al. 2016), the vast majority is currently in a mere prototypical state and not based on theoretical or empirical insights in these industries or organizations acting therein. Regrettably, very little research has empirically investigated the strategies and tactics applied by companies or entire industries when working on new blockchain solutions or acting on existing blockchains. As a mentionable exception, Beck and Müller-Bloch (2017) not only investigate value creation through blockchain technology but also outline a process of innovating based on blockchain technology in financial institutions. They show that management vision is of utmost importance in this process.

Beyond these valuable first steps, researchers have however largely ignored this field of research clearly connecting organization research and technology. Particularly blockchain technology's innovative character regarding distribution and its potential to interconnect potentially opportunistic actors within supply chains and entire industries yield many questions regarding strategy, tactics, and governance. For example, who holds which rights and power in industry-wide permissioned blockchain systems such as R3 in financial industry? How can existing inter-organizational business processes and value chains be redesigned given tamper-proof, distributed databases of transactions? Which factors determine whether firms interact more productively in an inter-organizational network based on blockchain technology, and how do different models of ensuring data privacy and confidentiality affect organizations' behavior? Lastly, research should also start to examine if, how, and why the purported new forms of organizations such as DAOs and DACs are viable and how such organizational forms can be effectively governed and be made compliant with legal regulations (Price 2016). All these questions are strongly related to traditional fields of IS and organizational research and therefore hold huge potential to expand extant research into the innovative field of blockchain technology.

In sum, theory-driven, empirical research has only recently started to address questions of managing and organizing actions of users and organizations in the face of blockchain systems. We expect enormous research contributions coming from IS and related disciplines during the next years.

\subsection{Avenues for Advanced Research}

Research should further address the important research directions we have pointed out so far. Table 4 should also help interested researchers to pick meaningful further research questions. In the following, we indicate interdisciplinary, theoretical and empirical linkages that will hopefully be particularly helpful for the closing of apparent research gaps.

\subsubsection{Potential for Multidisciplinary Research Collaborations}

Blockchain technology is pervasive in the sense that it introduces decentralization to the digital infrastructure spanning the layers from the hardware, over fabric and application layers up to the presentation layer (Glaser 2017). As the framework above shows, blockchain systems have the potential to affect various aspects of life due to their unique affordances. Both technological and application-oriented prospects promote input from and implications for other disciplines. However, as our review shows (Tables 2,3), extant publications still focus primarily on technological and business related topics and are often confined to the disciplines of computer science and 
Table 3 Overview of disciplines of blockchain related publications

\begin{tabular}{ll}
\hline Research discipline & Number of related publications \\
\hline Computer science & 28 \\
Information systems & 18 \\
Law & 9 \\
Finance & 6 \\
Political science & 5 \\
Others & 3 \\
\hline
\end{tabular}

information systems rather than addressing the broader societal, political or judicative questions. Only recently, major outlets in other disciplines have started picking up on the transformative potential of blockchain technology in their respective areas. For example, Yermack (2017) provides first concrete ideas how blockchain may influence practice as well as academic research of corporate governance and executive compensation. We argue that the practical and academic questions that emerge from blockchain technology and its design, application, and implications, provide a big potential for meaningful multidisciplinary research that extends beyond the boundaries of one specific discipline.

For design and features, we recommend considering related concepts and theories from computer science, law, and psychology to inform these research endeavors. As was shown by the literature review, a big proportion of the existent findings address this area of research. Particularly many conceptual papers and business-related technical improvements are published by computer scientists in their respective outlets (Table 3 ). Within the information systems discipline, the related design science research has successfully investigated first blockchain based use cases and first empirical projects are addressing adoption drivers and hindrances. We see a need for future research crossing the boundaries of computer science, information systems and law in many technical areas including cryptography (Yli-Huumo et al. 2016). For example, there is a big need for evaluating smart contracts through universal composability frameworks (Canetti 2001; Kosba et al. 2015) or non-interactive arguments of knowledge protocols (Juels et al. 2016). Where smart contracts indeed refer to business transactions, the expertise of scholars of law should not be neglected when designing technical solutions. Further advancements will pertain to developing means of moving proof of work protocols to proof of ownership models (Back 2017). However, as the technological development progresses and more applications become feasible, we expect findings from psychology to offer important input and contributions. Psychological research - and related work in information systems - has produced substantial insights regarding usability engineering (Dix 2009;
Shneiderman 2010) and adoption of information systems (Venkatesh and Davis 2000). In order to further advance blockchain technology dissemination, research will not only need to improve the ease-of-use but also investigate the perceived costs and benefits. First results, for example Fabian et al. (2016), suggest that questions of how users perceive and enact their privacy needs will need to be better understood in order to explain voluntary user adoption of blockchain solutions. We see substantial opportunities for collaborations across information systems and psychology to investigate such questions based on robust theory. Moreover, blockchain has been purported to allow trust-free transactions. Psychology based research can help determine whether and under which conditions this is actually the case. Theories on group decision making (e.g., group think or group polarization) from psychological research can inform the understanding of the decision formation process, for example, in the case of lacking consensus on a user level that leads to hard forks.

Moving towards measuring the value of blockchain and the costs at which these benefits come for the society and businesses will be a major driving force for the technological dissemination. The findings from this literature review support the concern recited by Glaser (2017) that blockchain is an innovative technology in search of use cases. We expect input from other disciplines like finance, economics, and sociology to support addressing this gap by offering insights on how to gain surplus and manage risks associated with the technology. We expect that multidisciplinary research will reveal, for example, whether and to which extent technically feasible blockchain based solutions [e.g., for notary services (Crosby et al. 2016)] will actually make it to productive applications compliant with legal requirements (Sean 2017). Finance is considered to be substantially affected by blockchain technology (Tapscott and Tapscott 2016). Thus, research on topics such as cryptofinance (Harvey 2016), securities issuance, insurance, trading and settlement will help advance terms of blockchain applicability (Nofer et al. 2017). While the financial market and respective intermediary institutions are currently in the focus of the debate, recent developments indicate that it is not necessarily the financial industry (McLannahan 2016) but other industries [e.g., logistics (Allison 2016)] that will be disrupted by the blockchain. Furthermore, economics can help to predict developments regarding the progression of blockchain based cryptocurrencies and derive means on how to integrate them into established currency systems. By transferring insights and principles identified by studying consequences of the networked economy (Choudary et al. 2016), economists will greatly contribute to the understanding of micro- and macroeconomic effects accompanying blockchain. In line with general digitalization 
developments (Loebbecke and Picot 2015), blockchain also poses challenges for the society. In collaboration with sociologists, research needs to provide political decision makers with reliable information regarding, for example, consequences for the job market and the consequences of enhanced transparency on the social behavior. Furthermore, currently discussed concepts such as cryptocitizens, cryptosustainability, and crypto-enlightened governance (Nichol 2016) will need to be properly elaborated in order to help understand and harness the societal effects of blockchain.

Ultimately, these insights on the technological and influential aspects of blockchain will help to inform the organization and management of blockchain related systems. Depending on the level of analysis, research will depend on collaborations with management, political sciences or law. When considering the handling of societal consequences and imposing regulations, knowledge from political sciences but also sociotechnical expertise from information systems will be imperative to providing impactful intelligence. Collaborating with law experts is going to advance research on the legislative aspects regarding intellectual properties and imposing legally binding frameworks for decentralized transactions of any type of goods thereby setting the boundaries for intermediary service providers (Vogel 2015). Scientists need to assess regulatory responses to crypto-currencies and draw useful lessons from regulatory deficiencies (Guadamuz and Marsden 2015). Furthermore, the potential empowerment of the individual at the expense of governmental power may even require a reevaluation of the principle of coercion as the basis for the rule of law and the eventual consequences for the balance between liberalism and democracy (Atzori 2015). Management sciences are important for deriving proper strategies on how to deploy blockchain services within the supply and value chains. Beck and Müller-Bloch (2017) already demonstrated the importance of top-level managers for introducing this novel technology within companies. To advance these insights towards actionable strategic advice for executives, insights from management science will help guide the process. However, we expect management contributions to go further by applying different theories, including for example transaction cost theory, to determine entrepreneurial consequences (Interlogica 2017; Williamson 2005).

Overall, we can conclude that the pervasiveness of the technology is currently not met by correspondingly comprehensive and multidisciplinary research approaches. This leaves great potential for future research to improve our understanding of the terms of change entailed by blockchain systems for the individual, businesses processes, and society at large. In Table 4 we depict several specific research questions derived from our previous analysis to provide guidance for future (partly interdisciplinary) blockchain research.

\subsubsection{Potential for Empirical Research}

Table 5 provides an overview of the methodological approaches taken in the papers we analyzed. Our analysis shows that there is a mentionable amount of conceptual and design-oriented research, particularly prototypes, and analytical investigations into cryptocurrencies. The amount of business-related quantitative research beyond cryptocurrencies is, however, extremely limited and theory-driven empirical research on blockchain related phenomena is generally scarce. On the one hand, this may be owed to the fact that blockchain technology is still relatively early in the hype cycle and researchers from outside computer science took long to realize the technology's potential. On the other hand, it may be owed to researchers' lack of knowledge about how to collect data for meaningful quantitative analyses in an area that has long been dominated by technical jargon and conceptual fuzziness (Glaser 2017). To enable more scholars to join this fruitful area of research, we briefly present some sources of data that may allow advancing business-related empirical research on blockchain technology and connect them to our framework and prior work.

For all levels of analysis presented in our framework, researchers can collect primary data for qualitative or quantitative analyses. For example, Beck and Müller-Bloch (2017) study the case of a firm in the financial industry based on interviews, Yuan and Wang (2016) present a case of a blockchain start-up for intermediating ride sharing, Abramova and Böhme (2016) and Fabian et al. (2016) conduct user surveys on Bitcoin, and Kazan et al. (2014) combine interview data from platform providers with archival news data. These approaches show that even the anonymity particular to some blockchain platforms does not prevent primary data collection. In fact, there are even successful examples of user surveys in clearly illegal markets fueled with cryptocurrency where participating subjects have to fear legal prosecution (Van Hout and Bingham 2013a, b). We consequently encourage fellow researchers to devise methods for collecting primary data despite the initial perception of obstacles related to the cryptographic aspects and network distribution involved in blockchain systems.

Blockchain systems consistently store a linear history of transactions. Although increasing data privacy and differentiating read and write permissions for transactions are two major contemporary research areas (Yli-Huumo et al. 2016), many blockchain implementations are currently fully transparent and all network actors can read their transactions and smart contracts. This has created quite 
Table 4 Exemplary blockchain research questions for future studies

\begin{tabular}{lll}
\hline Activities & Level of analysis & Selected research questions for future research \\
\hline Design and features & Users and society & How
\end{tabular}

Design and features

Platforms

Firms and industries

Measurement and value
Users and society

Intermediaries

Platforms

Firms and industries
How do specific blockchain induced affordances such as decentralization and different consensus mechanisms affect individual adoption?

How do traceability and potential deanonymization alter online transaction, investment, and spending behavior?

How can smart contracts and the services they provide interoperate across multiple blockchains?

Are some smart contracts particularly suited to be hosted in certain blockchain environments?

How can interfaces between smart contracts and existing information systems be designed to increase interoperability?

What are the technological interdependencies between different blockchain features (e.g., levels of permission and consensus mechanisms)?

How can the technical strengths of multiple public and private blockchain platforms be combined for complex business transactions?

Which combination of blockchain features offers greatest protection against issues such as $50+1$ attacks?

Which features (e.g. consensus mechanisms) make a permissionless blockchain applicable for different company use cases?

How can scalability issues be overcome in order to enable Internet of Things transactions?

How can business processes involving sensitive data such as patient information or financial records be implemented on blockchain?

Do the blockchain provided benefits of immutability and decentralized control translate into monetary value?

Do features like the perceived transaction speed and control over money flow affect the individual willingness to pay?

Which new forms of employment arise for trained experts of intermediary services companies in a blockchain industry?

To what extent does trust in an algorithm differ from trust in a third-party service provider?

How can fraudulent coin offerings be detected and legally indicted?

Does the relationship between token transfer and value follow the same principals as trading volume and price?

Does the removal of an intermediary party cause an in- or decrease in the perceived empowerment and control?

Does the combination of currency and service offering within the same blockchain enable new forms of dynamic pricing?

Can transaction traceability be leveraged to identify criminals or prevent unlawful transactions on darknet markets?

What are the (dis-)advantages of traditional auditing systems compared to blockchain based corporate auditing?

How can decentralized blockchain systems help overcome issues of fragmented markets?

How can tax authorities utilize transaction logging to automate tax deductions and avoid tax fraud?

To what extent does blockchain enabled decentralization and traceability challenge sharing economy platforms such as Uber and AirBnB?

To what extent are the consequences of automated decentralized intermediation for service providers comparable to those of the industrial revolution for manufacturers?

How does blockchain enabled traceability within supply chains affect product prices and quality?

Which kinds of business models can be economically successful in a blockchain industry? 
Table 4 continued

\begin{tabular}{|c|c|c|}
\hline Activities & Level of analysis & Selected research questions for future research \\
\hline \multirow[t]{14}{*}{ Management and organization } & \multirow[t]{5}{*}{ Users and society } & $\begin{array}{l}\text { How can blockchain based voting mechanisms mitigate threats of group decision- } \\
\text { making biases? }\end{array}$ \\
\hline & & $\begin{array}{l}\text { How can blockchain technology increase participation of citizens in political decision- } \\
\text { making? }\end{array}$ \\
\hline & & How do DAO/DAC structures affect the influence of individual stakeholders? \\
\hline & & $\begin{array}{l}\text { Which insights from political referendums can be transferred to DAO/DAC decision } \\
\text { making? }\end{array}$ \\
\hline & & $\begin{array}{l}\text { What is the individually preferred balance between legal blockchain regulation and } \\
\text { operational risk? }\end{array}$ \\
\hline & \multirow[t]{3}{*}{ Intermediaries } & $\begin{array}{l}\text { How do differences in ecosystem governance affect the provision of services on public } \\
\text { blockchains? }\end{array}$ \\
\hline & & $\begin{array}{l}\text { Which variations of token functionalities (such as representations of property, utility, or } \\
\text { rewards) are most conducive to disintermediation? }\end{array}$ \\
\hline & & $\begin{array}{l}\text { How does outsourcing to blockchain smart contracts differ from traditional outsourcing } \\
\text { regarding contract completeness and governance mechanisms? }\end{array}$ \\
\hline & \multirow[t]{3}{*}{ Platforms } & $\begin{array}{l}\text { Which consensus mechanisms can blockchain platforms deploy to avoid } \\
\text { monopolization of power? }\end{array}$ \\
\hline & & $\begin{array}{l}\text { What are the economic consequences of managerial interventions on public blockchains } \\
\text { such as hard forks? }\end{array}$ \\
\hline & & $\begin{array}{l}\text { How can blockchain platforms device community mechanisms to facilitate protocol } \\
\text { evolution and prevent forks? }\end{array}$ \\
\hline & \multirow[t]{3}{*}{ Firms and industries } & $\begin{array}{l}\text { How can companies meet international data privacy standards when conducting } \\
\text { blockchain-based transactions? }\end{array}$ \\
\hline & & $\begin{array}{l}\text { Which forms of consensus mechanisms should companies deploy when conducting } \\
\text { industry-wide decentralized transactions? }\end{array}$ \\
\hline & & $\begin{array}{l}\text { Who finances and governs the development and operation of decentralized inter- } \\
\text { organizational blockchain systems? }\end{array}$ \\
\hline
\end{tabular}

Table 5 Overview of methodologies of blockchain related publications

\begin{tabular}{lll}
\hline Paradigm & Study methodology & Number of related publications \\
\hline Conceptual and design-oriented & Conceptual & 25 \\
& Design Science/prototyping & 17 \\
& Literature review & 8 \\
Theory-driven empirical & Case study & 6 \\
& Simulation & 5 \\
& Survey & 4 \\
Unclear & Experiment & 1 \\
& Others & 3 \\
\hline
\end{tabular}

mentionable opportunities to collect and analyze secondary data by inspecting publicly available blockchain systems including Bitcoin and Ethereum. For technical questions, researchers have already made use of these possibilities (Tschorsch and Scheuermann 2016; Yli-Huumo et al. 2016), but little business-related research has done so. While some researchers may want to analyze public blockchains fully on their machines to apply or develop specific measures, for example for social network analysis (Glaser et al. 2014; Maesa et al. 2016), others may want to rely at least partly on aggregation services that can be found on the internet. As such, Cocco and Marchesi (2016) use blockchain.info to calibrate their analytical model. There are several such online services and blockchain analytics software solutions that allow for pre-analyzing or fully analyzing data from different public blockchain systems. Several provide application programming interfaces (APIs) to directly export and consume data. In the appendix, Table A2, we summarize several popular data providers and depict some characteristic pieces of data that can be retrieved via each provider. Detailed explanations for each piece of data can be found on the websites and 
documentations of the APIs and are omitted here for the sake of conciseness.

We argue that such data providers and blockchain analytics services can fruitfully be used for empirical research and save researchers some trouble of going deep into protocols of each blockchain implementation. Particularly research on platform and on user levels may be interested in using such services as those are, together with single transactions, the levels of analysis that are typically provided by the services. For research on the societal level, we moreover suggest that researchers may want to analyze the points where public blockchains and the physical world interface. As such, the geographic distribution of ATMs exchanging Bitcoin to fiat currency may be one interesting starting point and is provided publicly (see Table A2). For studies on intermediaries and smart contracts providing intermediary services, the website ether.camp may be a starting point with rudimentary analytical capabilities for smart contracts on Ethereum. Lastly, researchers may simply be interested in finding representative companies for their firm level studies. For this purpose, Table A2 also depicts a service that ranks blockchain companies and consortia by their activity on social media, which can be used to gain a first overview of relevant candidate firms.

In sum, we hope that these data sources provide valuable starting points for projects of researchers who want to become active in empirically investigating business-relevant phenomena related to blockchain technology.

\section{Discussion}

This paper set out to chart the state of knowledge on blockchain technology beyond cryptocurrencies and to identify current as well as prospective research topics to enable meaningful scholarly engagement in blockchain research. The intent is to streamline and inform future blockchain related scientific endeavors across disciplines to advance insights in terms of blockchain application. The insights provided by the literature review in combination with the adapted framework for blockchain research have a number of implications for research.

First and foremost, there is a dominant concentration of extant work on design and features of blockchain technology that is largely driven by conceptual, prototyping, and analytical papers, often on cryptocurrencies. This is consistent with prior reviews on blockchain from a more technical perspective (Morisse 2015; Tschorsch and Scheuermann 2016; Yli-Huumo et al. 2016). To further understand the applicability, use and effects of blockchain technology, we propose that future research should sophisticatedly consider interdependencies and trade-offs between different blockchain features (e.g., scalability, security and privacy) as well as the effects of the separate features on the different levels of analysis (Anceaume et al. 2016; Walsh et al. 2016; Yli-Huumo et al. 2016). Despite the purported disruptive potential and the grand expectations about blockchains, the almost exclusive focus on technology has led to a situation where critically needed research has largely been neglected (Glaser 2017). Our review shows that this holds true for research on value creation and measurement as well as governance and management of blockchain systems, which encompass organizational and individual users. The current application focus of blockchain on the financial market (i.e., stock exchange, banks, credit card companies, and cryptocurrencies) can be explained by the Bitcoin background of the technology and the general orientation of financial institutions towards digital services. However, the pervasiveness and extent of the technology call for considering areas of application beyond the currently discussed financial market [e.g., logistics, procurement (Allison 2016; Nofer et al. 2017; Price 2015)]. The current state of research suggests particular values of blockchain for supply chain management (Korpela et al. 2017) through the combination with IoT services (Christidis and Devetsikiotis 2016) or transmission technologies [e.g., RFID (Feng 2016)]. Regarding societal and legal consequences, some forms of research have rapidly developed [e.g., essays on regulatory issues regarding cryptocurrencies (Guadamuz and Marsden 2015)], whereas other aspects are barely considered (e.g., decision making mechanisms, rule enforcement, coercion). Despite the heightened expectations regarding the empowerment of the individual as opposed to companies or the government, critical analyses of the applicability of blockchain for societal purposes (e.g., in e-government) emphasize that this can by no means be seen as a development towards dispensability of state control (Atzori 2015). Thus, we assume that research on measurement and value as well as management and organization that builds upon comprehensive insights on blockchain design and features can provide essential contributions regarding the terms of blockchain application.

Second, our analysis revealed that there are only scarce examples of empirical and theory-driven research. Although we cannot claim to know the underlying reasons, the presented research agenda in combination with the starting points for empirical investigations are intended to support researchers in conducting rigorous research in this highly relevant area.

Third, analyzing contributions of the distinct disciplines revealed that there is little multidisciplinary research to reflect the ramifications of blockchain systems that extend far beyond technological issues into economy and society. We are convinced that collaborations across disciplinary borders are fruitful and actually necessary for meaningful 
research on blockchain systems. First scholars from multiple disciplines have begun to examine single technical features to build an informed understanding that enables legislators and policymakers to address regulatory concerns (Kiviat 2015). Joining their forces on the outlined open questions should, from our perspective, benefit the comprehensiveness of their important research projects. Thus, we have introduced several important areas and open questions where multidisciplinary research is critically needed (e.g., group decision-making, cryptocitizens, coercion). At the same time, we acknowledge that multidisciplinary research is challenging regarding the selection of proper publication outlets and the proper research scope under consideration of the targeted discipline. Thus, while multidisciplinary research poses great challenges, we expect this will be the way to cope with the implications of blockchain technology and to inform society, industry and academia how to shape the technology to leverage the particular prospective benefits.

\section{Conclusion}

Blockchain technology is among the most trending technologies and is said to have strong disruptive potential (Gartner 2016). At the same time, however, blockchain is commonly referred to as an innovative technology in search of use cases (Glaser 2017) and may possibly not fulfill the great expectations placed on it (Avital et al. 2016). We assume that a comprehensive overview of the present scientific research activities in a framework with prospective guidelines for future research will help to sustain blockchain research beyond the current hype. Addressing this objective, we created a general research framework for blockchain systems based on a popular and successful template (Aral et al. 2013) and the technological affordances of blockchain technology (Glaser 2017). It draws attention to the questions how different blockchain systems should be designed, how blockchains can be deployed to generate value, and how blockchain systems including organizational, individual, and artificial actors can be managed and governed. These general questions relate to more specific ones on different levels of analysis, namely for users and society, intermediaries, platforms, as well as firms and industries. Reviewing and classifying the existent literature into the respective areas, we identified the predominant and the neglected fields of blockchain research beyond cryptocurrencies. By providing online access to the current state-of-knowledge and inviting researchers to collaborate by submitting new blockchain publications, we intend to substantially inform future research. ${ }^{3}$ This study also highlighted the intersections of different disciplines that provide the basis for multidisciplinary research collaboration to create meaningful advances in blockchain research. Lastly, we provided an overview of potential data sources for investigations on different levels of analysis to help scholars get started with more empirical research. Our findings suggest that published research provides a decent understanding of the current technological state-of-practice. Investigations into consequences of different technological variations, into the business value of blockchain systems, and into their management and organization are fairly scarce. We conclude by urging researchers to take on the challenge and achieve contributions that advance the general knowledge on blockchain systems, particularly regarding value creation and management. Conceptually, we contribute to blockchain research by providing a prospective research framework that was adapted from the prominent guiding agenda for a disruptive network technology by Aral et al. (2013). Even beyond the research questions defined in this paper, the conceptual framework can be used to map focal user activities (Design, Measure, Manage) and levels of analysis (Users, Intermediaries, Platforms, Firms) in order to spot open areas for research in the future or systematically create new research questions. The combination of categories is intended to guide research and structure findings. Therefore, these categories are not set to be mutually exclusive. Studies can focus on one activity that simultaneously addresses different levels of analysis [e.g., Design and Features: Intermediaries, Firms and Industries (Juels et al. 2016)] or pursue different activities targeting similar levels of analysis [e.g., Platforms: Design and Features, Management and Organizations (Luu et al. 2015)]. Acknowledging the bigger picture by referring to an established framework will hopefully also allow future researchers to comprehensively guide investigations beyond areas mentioned by the current literature like existent reviews do (e.g., Yli-Huumo et al. 2016).

The contributions of the study need to be considered in the light of its limitations. Due to the emergent nature of the topic, the reviewed literature was not published in highranking journals with prolonged review cycles. Therefore, parts of the developed research questions are based on the exchange with experienced blockchain developers and other precarious sources. Nonetheless, the key components of the work and predominant share of literature were drawn from peer-reviewed outlets in the information systems and computer science disciplines representing the current state of knowledge. Furthermore, the goal of this work was to develop a framework of blockchain research as a whole. Therefore, the share of Bitcoin literature is quantitatively

\footnotetext{
3 We provide open access to the overview of current scientific knowledge (Table 2) here: http://bit.ly/BCSOTA.
} 
underrepresented. We refer to the existing reviews on this specific type of blockchain (Glaser et al. 2014; Morisse 2015; Tschorsch and Scheuermann 2016; Wörner et al. 2016), while incorporating the general insights into this review.

\section{References}

Abramova S, Böhme R (2016) Perceived benefit and risk as multidimensional determinants of bitcoin use: a quantitative exploratory study. In: 37th International conference on information systems, Dublin

Agarwal R, Tiwana A (2015) Editorial—evolvable systems: through the looking glass of IS. Inf Syst Res 26(3):473-479

Ainsworth RT, Shact A (2016) Blockchain (distributed ledger technology) solves VAT fraud. Boston Univ Law Econ Res Pap 41(16):1-25

Aitzhan NZ, Svetinovic D (2016) Security and privacy in decentralized energy trading through multi-signatures, blockchain and anonymous messaging streams. IEEE Trans Dependable Secure Comput 99:1-14

Allison I (2016) Shipping giant Maersk tests blockchain-powered bill of lading. Int Bus Times. http://www.ibtimes.co.uk/shippinggiant-maersk-tests-blockchain-powered-bills-lading-1585929. Accessed 7 Jan 2017

Anceaume E, Lajoie-Mazenc T, Ludinard R, Sericola B (2016) Safety analysis of bitcoin improvement proposals. In: 15th International symposium on network computing and applications (NCA). IEEE, pp 318-325

Aral S, Dellarocas C, Godes D (2013) Introduction to the special issue-social media and business transformation: a framework for research. Inf Syst Res 24(1):3-13

Atzori M (2015) Blockchain technology and decentralized governance: is the state still necessary? SSRN Working Paper

Avital M, Beck R, King J, Rossi M, Teigland R (2016) Panel on: jumping on the blockchain bandwagon: lessons of the past and outlook to the future. In: 37th International conference on information systems, Dublin

Azaria A, Ekblaw A, Vieira T, Lippman A (2016) MedRec: using blockchain for medical data access and permission management. In: 2nd International conference on open and big data (OBD), Vienna. IEEE, pp 25-30

Back A (2017) Ethereum to switch to "proof of stake" protocol despite skepticism. Disruptive.asia. https://disruptive.asia/ether eum-proof-stake-protocol/. Accessed 18 April 2017

Back A, Corallo M, Dashjr L, Friedenbach M, Maxwell G, Miller A, Poelstra A, Timón J, Wuille P (2014) Enabling blockchain innovations with pegged sidechains. Blockstream. http://www. blockstream.com/sidechains.pdf. Accessed 15 Dec 2016

Banafa A (2016) How to secure the internet of things (IoT) with blockchain. Datafloq. https://www.datafloq.com/read/securinginternet-of-things-iot-with-blockchain/2228. Accessed 7 Jan 2017

Beck R, Müller-Bloch C (2017) Blockchain as radical innovation: a framework for engaging with distributed ledgers. In: 50th Hawaii international conference on system sciences, Waikoloa

Beck R, Stenum Czepluch J, Lollike N, Malone S (2016) Blockchainthe gateway to trust-free cryptographic transactions. In: 24th European conference on information systems, İstanbul

Bell TW (2016) Copyrights, privacy, and the blockchain. Ohio North Univ Law Rev 42(16):1-33
Boell SK, Cecez-Kecmanovic D (2014) A hermeneutic approach for conducting literature reviews and literature searches. Commun Assoc Inf Syst 34(1):257-286

Brandon D (2016) The blockchain: the future of business information systems? Int J Acad Bus World 10(2):33-40

Brenig C, Schwarz J, Rückeshäuser N (2016) Value of decentralized consensus systems-evaluation framework. In: 24th European conference on information systems (ECIS), İstanbul

Buterin V (2014a) A next-generation smart contract and decentralized application platform. Ethereum. https://www.ethereum.org/pdfs/ EthereumWhitePaper.pdf. Accessed 15 Dec 2016

Buterin V (2014b) Scalability, part 3: on metacoin history and multichain. Ethereum Blog, Technical. https://blog.ethereum. org/2014/11/13/scalability-part-3-metacoin-history-multichain/. Accessed 15 Dec 2016

Buterin V (2015) On public and private blockchains. Ethereum blog, crypto renaissance salon. https://blog.ethereum.org/2015/08/07/ on-public-and-private-blockchains/. Accessed 15 Dec 2016

Canetti R (2001) Universally composable security: a new paradigm for cryptographic protocols. In: 42nd IEEE symposium on foundations of computer science. IEEE, pp 136-145

Caytas JD (2016) Developing blockchain real-time clearing and settlement in the EU, US, and globally. Columbia J Europ Law. http://cjel.law.columbia.edu/preliminary-reference/2016/develop ing-blockchain-real-time-clearing-and-settlement-in-the-eu-u-sand-globally-2/. Accessed 15 Dec 2016

Choudary SP, Van Alstyne MW, Parker GG (2016) Platform revolution: how networked markets are transforming the economy-and how to make them work for you. Norton, New York

Christidis K, Devetsikiotis M (2016) Blockchains and smart contracts for the internet of things. IEEE Access 4:2292-2303

Cocco L, Marchesi M (2016) Modeling and simulation of the economics of mining in the bitcoin market. PLoS One 11(10): 0164603

Coleman L (2016) Ethereum responds to recent DDoS attack. Cryptocoins news. https://www.cryptocoinsnews.com/ether eum-responds-to-recent-ddos-attack/. Accessed 7 Jan 2017

Crosby M, Pattanayak P, Verma S, Kalyanaraman V (2016) Blockchain technology: beyond bitcoin. Appl Innov 2:6-10

Danezis G, Meiklejohn S (2016) Centrally banked cryptocurrencies. In: Network and distributed system security symposium, San Diego

De Filippi P (2016) The interplay between decentralization and privacy: the case of blockchain technologies. J Peer Prod 9:1-19

Decker C, Wattenhofer R (2013) Information propagation in the bitcoin network. In: IEEE thirteenth international conference on peer-to-peer computing, Trento. IEEE, pp 1-10

Dennis R, Owen G (2015) Rep on the block: a next generation reputation system based on the blockchain. In: 10th international conference for internet technology and secured transactions (ICITST), London. IEEE, pp 131-138. https://doi.org/10.1109/ ICITST.2015.7412073

Detrixhe J (2016) Scotland to start own stock exchange using blockchain technology. Bloomberg. https://www.bloomberg. com/news/articles/2016-10-27/scotland-to-start-own-stockexchange-using-blockchain-technology. Accessed 6 Jan 2017

Dix A (2009) Human-computer interaction. In: Liu L, Özsu MT (eds) Encyclopedia of database systems. Springer, New York, pp 1327-1331

Dwyer GP (2015) The economics of bitcoin and similar private digital currencies. J Financial Stab 17:81-91

Fabian B, Ermakova T, Sander U (2016) Anonymity in bitcoin? The users' perspective. In: 37th International conference on information systems (ICIS), Dublin

Feng T (2016) An agri-food supply chain traceability system for China based on RFID \& blockchain technology. In: 13th 
International conference on service systems and service management (ICSSSM), Kunming. IEEE, pp 1-6

Finley K (2016) Here's how IBM is planning to use its own blockchain software. Wired. https://www.wired.com/2016/06/ heres-ibm-planning-use-blockchain-software/. Accessed 6 Jan 2017

Fujimura S, Watanabe H, Nakadaira A, Yamada T, Akutsu A, Kishigami JJ (2015) BRIGHT: a concept for a decentralized rights management system based on blockchain. In: IEEE 5th International conference on consumer electronics, Berlin. IEEE, pp 345-346

Gartner (2016) Gartner's 2016 hype cycle for emerging technologies identifies three key trends that organizations must track to gain competitive advantage. Gartner. http://www.gartner.com/news room/id/3412017. Accessed 10 Jan 2017

Gervais A, Karame GO, Wüst K, Glykantzis V, Ritzdorf H, Capkun S (2016) On the security and performance of proof of work blockchains. In: 2016 ACM SIGSAC conference on computer and communications security, Vienna. ACM, pp 3-16

Gipp B, Kosti J, Breitinger C (2016) Securing video integrity using decentralized trusted timestamping on the bitcoin blockchain. In: 10th Mediterranean conference on information systems, Paphos

Glaser F (2017) Pervasive decentralisation of digital infrastructures: a framework for blockchain enabled system and use case analysis. In: 50th Hawaii international conference on system sciences (HICSS 2017), Waikoloa

Glaser F, Bezzenberger L (2015) Beyond cryptocurrencies-a taxonomy of decentralized consensus systems. In: 23rd European conference on information systems, Münster

Glaser F, Zimmermann K, Haferkorn M, Weber MC, Siering M (2014) Bitcoin-asset or currency? Revealing users' hidden intentions. In: 22nd European conference on information systems (ECIS), Tel Aviv

Guadamuz A, Marsden C (2015) Blockchains and bitcoin: regulatory responses to cryptocurrencies. First Monday 20(12). https://doi. org/10.5210/fm.v20i12.6198

Harvey CRH (2016) Cryptofinance. SSRN. https://doi.org/10.2139/ ssrn.2438299

Hashemi SH, Faghri F, Rausch P, Campbell RH (2016) World of empowered IoT users. In: IEEE first international conference on internet-of-things design and implementation (IoTDI), Berlin. IEEE, pp 13-24

Hayes A (2016) Decentralized banking: monetary technocracy in the digital age. In: 10th Mediterranean conference on information systems (MCIS), vol 3. Paphos. AIS

Holotiuk F, Pisani F, Moormann J (2017) The impact of blockchain technology on business models in the payments industry. In: 13th International conference on wirtschaftsinformatik, St. Gallen. AIS, pp 912-926

Hoofnagle CJ, King J, Li S, Turow J (2010) How different are young adults from older adults when it comes to information privacy attitudes and policies? SSRN 1589864

Hsieh H-F, Shannon SE (2005) Three approaches to qualitative content analysis. Qual Health Res 15(9):1277-1288

Interlogica (2017) The socio-economic effects of the blockchain. Interlogica. http://www.interlogica.it/en/insight/blockchainsocio-economic-effects/. Accessed 18 Apr 2017

Juels A, Kosba A, Shi E (2016) The ring of gyges: using smart contracts for crime. In: SIGSAC conference on computer and communications security, Vienna, pp 283-295

Kazan E, Tan C-W, Lim ET (2014) Towards a framework of digital platform disruption: a comparative study of centralized \& decentralized digital payment providers. In: 25th Australasian conference on information systems, Auckland
King NJ, Raja VT (2012) Protecting the privacy and security of sensitive customer data in the cloud. Comput Law Secur Rev 28(3):308-319

Kitchenham B, Charters S (2007) Guidelines for performing systematic literature reviews in software engineering. EBSE Technical Report EBSE-2007-01

Kiviat T (2015) Beyond bitcoin: issues in regulating blockchain transactions. Duke Law J 65(3):569-608

Korpela K, Hallikas J, Dahlberg T (2017) Digital supply chain transformation toward blockchain integration. In: 50th Hawaii international conference on system sciences, Manoa

Kosba A, Miller A, Shi E, Wen Z, Papamanthou C (2015) Hawk: the blockchain model of cryptography and privacy-preserving smart contracts. Paper presented at the 37th IEEE symposium on security and privacy, San Jose

Kraft D (2016) Difficulty control for blockchain-based consensus systems. Peer-to-Peer Netw Appl 9(2):397-413

Krippendorff K (2012) Content analysis: an introduction to its methodology, 3rd edn. Sage, Thousand Oaks

Lee L (2016) New kids on the blockchain: how bitcoin's technology could reinvent the stock market. Hastings Bus Law J 12(2):138

Lee J-H, Pilkington M (2017) How the blockchain revolution will reshape the consumer electronics industry. IEEE Consum Electron Mag 6(3):19-23

Lewenberg Y, Sompolinsky Y, Zohar A (2015) Inclusive block chain protocols. In: International conference on financial cryptography and data security, San Juan, Springer, pp 528-547

Lindman J, Tuunainen VK, Rossi M (2017) Opportunities and risks of blockchain technologies-a research agenda. In: 50th Hawaii international conference on system sciences, Manoa, pp 1-10

Loebbecke C, Picot A (2015) Reflections on societal and business model transformation arising from digitization and big data analytics: a research agenda. J Strateg Inf Syst 24(3):149-157

Lotti L (2016) Contemporary art, capitalization and the blockchain: on the autonomy and automation of art's value. Financ Soc 2(2):96-110

Lowry PB, Zhang J, Wang C, Siponen M (2016) Why do adults engage in cyberbullying on social media? An integration of online disinhibition and deindividuation effects with the social structure and social learning model. Inf Syst Res 27(4):962-986

Luu L, Teutsch J, Kulkarni R, Saxena P (2015) Demystifying incentives in the consensus computer. In: Proceedings of the 22nd ACM SIGSAC conference on computer and communications security, ACM, pp 706-719

Maesa DDF, Marino A, Ricci L (2016) Uncovering the bitcoin blockchain: an analysis of the full users graph. In: 3rd IEEE international conference on data science and advanced analytics (DSAA), Montreal. IEEE, pp 537-546

Mainelli M, Smith M (2015) Sharing ledgers for sharing economies: an exploration of mutual distributed ledgers (aka blockchain technology). J Financ Perspect 3(3):38-69

McJohn SM, McJohn I (2016) The commercial law of bitcoin and blockchain transactions. Uniform Commer Code Law J 16(13). https://ssrn.com/abstract=2874463

McLannahan B (2016) Goldman Sachs quits R3 blockchain consortium. Financial times. https://www.ft.com/content/598934e0b010-11e6-9c37-5787335499a0. Accessed 7 Jan 2017

Meiklejohn S, Pomarole M, Jordan G, Levchenko K, McCoy D, Voelker GM, Savage S (2013) A fistful of bitcoins: characterizing payments among men with no names. In: Proceedings of the 2013 conference on Internet measurement conference. ACM, pp 127-140

Mettler M (2016) Blockchain technology in healthcare: the revolution starts here. In: IEEE 18th international conference on e-health networking, applications and services, Munich. IEEE, pp 1-3 
Morini M (2016) From 'blockchain hype' to a real business case for financial markets. J Financ Transform 45:30-40

Morisse M (2015) Cryptocurrencies and bitcoin: charting the research landscape. In: 21st Americas conference on information systems, Fajardo

Morris R (1994) Computerized content analysis in management research: a demonstration of advantages \& limitations. J Manag 20(4):903-931

Nakamoto S (2008) Bitcoin: a peer-to-peer electronic cash system. https://bitcoin.org/bitcoin.pdf. Accessed 15 Dec 2016

Nguyen QK (2016) Blockchain-a financial technology for future sustainable development. In: 3rd International conference on green technology and sustainable development (GTSD), Kaohsiung, IEEE, pp 51-54

Nichol PB (2016) Healthcare interoperability research propositions of the ONC blockchain challenge. CIO.com. https://www.scphca. org/media/122448/scphca_blockchain_presentation_official. pdf. Accessed 18 Apr 2017

Nofer M, Gomber P, Hinz O, Schiereck D (2017) Blockchain. Bus Inf. Syst Eng 59(3):183-187

Ølnes S (2016) Beyond bitcoin enabling smart government using blockchain technology. In: International conference on electronic government and the information systems perspective. Springer, pp 253-264

Paech P (2016) Securities, intermediation and the blockchain: an inevitable choice between liquidity and legal certainty? Uniform Law Rev 21(4):1-33

Paré G, Trudel M-C, Jaana M, Kitsiou S (2015) Synthesizing information systems knowledge: a typology of literature reviews. Inf Manag 52(2):183-199

Peters GW, Panayi E, Chapelley A (2015) Trends in cryptocurrencies and blockchain technologies: a monetary theory and regulation perspective. J Financ Perspect 3(3):92-113

Pilkington M, Crudu R, Grant LG (2017) Blockchain and bitcoin as a way to lift a country out of poverty-tourism 2.0 and e-governance in the Republic of Moldova. Int $\mathrm{J}$ Internet Technol Secured Trans 7(2):115-143

Price R (2015) This London startup could make diamond theft a thing of the past-and that's just the start. Bus Insider. http://www. businessinsider.com/everledger-ledger-diamonds-blockchaintech-theft-fraud-2015-8?r=UK\&IR=T. Accessed 18 Apr 2017

Price R (2016) Digital currency Ethereum is cratering because of a $\$ 50$ million hack. Bus Insider. http://www.businessinsider.de/ dao-hacked-ethereum-crashing-in-value-tens-of-millions-alleg edly-stolen-2016-6?r=UK\&IR=T. Accessed 6 Jan 2017

Puschmann T, Alt R (2016) Sharing economy. Bus Inf Syst Eng 58(1):93-99

Raskin M (2016) The law of smart contracts. Georgetown Law Technol Rev 304(1):1-37

Reyes CL (2016) Moving beyond bitcoin to an endogenous theory of decentralized ledger technology regulation: an initial proposal. Villanova Law Rev 61(1):181-228

Reyes CL (2017) Conceptualizing cryptolaw. Nebraska, Law Rev, p 96

Ron D, Shamir A (2013) Quantitative analysis of the full bitcoin transaction graph. In: International conference on financial cryptography and data security. Springer, pp 6-24

Rosenfeld M (2012) Overview of colored coins. https://bitcoil.co.il/ BitcoinX.pdf. Accessed 15 Dec 2016

Rourke L, Anderson T, Garrison DR, Archer W (2001) Methodological issues in the content analysis of computer conference transcripts. Int J Artif Intell Educ 12:8-22

Rückeshäuser N (2017) Do we really want blockchain-based accounting? Decentralized consensus as enabler of management override of internal controls. In: 13th International conference on Wirtschaftsinformatik, St. Gallen. AIS, pp 16-30
Sanda T, Inaba H (2016) Proposal of new authentication method in Wi-Fi access using bitcoin 2.0. In: IEEE 5th global conference on consumer electronics, Kyoto. IEEE, pp 1-5

Savelyev A (2017) Contract law 2.0: 'smart' contracts as the beginning of the end of classic contract law. Inf Commun Technol Law 26(2):116-134

Schwartz D, Youngs N, Britto A (2014) The ripple protocol consensus algorithm. https://ripple.com/files/ripple_consensus_ whitepaper.pdf. Accessed 15 Dec 2016

Science UGOf (2016) Distributed ledger technology: beyond block chain. https://www.gov.uk/government/uploads/system/uploads/ attachmentldata/file/492972/gs-16-1-distributed-ledger-technol ogy.pdf. Accessed 15 Dec 2016

Sean (2017) Does notarization on the blockchain actually work? Decentralize today. https://decentralize.today/does-notarizationon-the-blockchain-actually-work-d8006443c0b9?gi= 169dd5363077. Accessed 19 Apr 2017

Shackelford S, Myers S (2016) Block-by-block: leveraging the power of blockchain technology to build trust and promote cyber peace. Yale J Law Technol 85(16):1-55

Shin L (2016) Looking to integrate blockchain into your business? Here's how. Forbes. http://www.forbes.com/sites/laurashin/ 2016/05/10/looking-to-integrate-blockchain-into-your-businessheres-how/\#3f1dc56d9182. Accessed 7 Jan 2017

Shirky C (2011) The political power of social media. Foreign Aff 90(1):28-41

Shneiderman B (2010) Designing the user interface: strategies for effective human-computer interaction. Pearson Education India, Delhi, India

Sikorski JJ, Haughton J, Kraft M (2017) Blockchain technology in the chemical industry: machine-to-machine electricity market. Appl Energy 195:234-246

Stewart KJ, Gosain S (2006) The impact of ideology on effectiveness in open source software development teams. MIS Q 30(2):291-314

Szabo N (1997) Formalizing and securing relationships on public networks. First Monday 2(9). https://doi.org/10.5210/fm.v2i9. 548

Tapscott D, Tapscott A (2016) The impact of the blockchain goes beyond financial services. Harvard business review. https://hbr. org/2016/05/the-impact-of-the-blockchain-goes-beyond-finan cial-services. Accessed 7 Jan 2017

Tschorsch F, Scheuermann B (2016) Bitcoin and beyond: a technical survey on decentralized digital currencies. IEEE Commun Surv Tutor 18(3):2084-2123

Van Hout MC, Bingham T (2013a) 'Silk Road', the virtual drug marketplace: a single case study of user experiences. Int J Drug Policy 24(5):385-391

Van Hout MC, Bingham T (2013b) 'Surfing the Silk Road': a study of users' experiences. Int J Drug Policy 24(6):524-529

Venkatesh V, Davis FD (2000) A theoretical extension of the technology acceptance model: four longitudinal field studies. Man Sci 46(2):186-204

Vernon T (2016) 5 ways blockchain will transform financial services. Finextra. https://www.finextra.com/blogposting/13068/5-waysblockchain-will-transform-financial-services. Accessed 7 Jan 2017

Vogel N (2015) The great decentralization: how Web 3.0 will weaken copyrights. John Marshall Rev Intell Prop Law 15(1):6-31

Walch A (2017) The path of the blockchain lexicon (and the law). Rev Bank Financ Law 36:1-37

Walsh C, OReilly P, Gleasure R, Feller J, Li S, Cristoforo J (2016) New kid on the block: a strategic archetypes approach to understanding the blockchain. In: 37th International conference on information systems, Dublin 
Watanabe H, Fujimura S, Nakadaira A, Miyazaki Y, Akutsu A, Kishigami JJ (2015) Blockchain contract: a complete consensus using blockchain. In: IEEE 4th global conference on consumer electronics, Osaka. IEEE, pp 577-578

Watanabe H, Fujimura S, Nakadaira A, Miyazaki Y, Akutsu A, Kishigami J (2016) Blockchain contract: securing a blockchain applied to smart contracts. In: IEEE International conference on consumer electronics, Las Vegas. IEEE, pp 467-468

Webster J, Watson RT (2002) Analyzing the past to prepare for the future: writing a literature review. MIS Q 26(2):xiii-xxiii

Williamson OE (2005) Transaction cost economics. In: Menard C, Shirley MM (eds) Handbook of new institutional economics. Springer, New York, pp 41-65

Wood G (2014) Ethereum: a secure decentralised generalised transaction ledger. Gavwood, vol 2017, http://gavwood.com/ Paper.pdf. Accessed 15 Dec 2016

Wörner D, Von Bomhard T, Schreier Y-P, Bilgeri D (2016) The bitcoin ecosystem: disruption beyond financial services? In: 24th European conference on information systems, İstanbul

Xu X, Pautasso C, Zhu L, Gramoli V, Ponomarev A, Tran AB, Chen $S$ (2016) The blockchain as a software connector. In: 13th Working IEEE/IFIP conference on software architecture, Venice. IEEE, pp 182-191

Xu X, Weber I, Staples M, Zhu L, Bosch J, Bass L, Pautasso C, Rimba P (2017) A taxonomy of blockchain-based systems for architecture design. In: 2017 IEEE international conference on software architecture (ICSA), Gothenburg. IEEE, pp 243-252
Yasin A, Liu L (2016) An online identity and smart contract management system. In: IEEE 40th annual computer software and applications conference, Atlanta. IEEE, pp 192-198

Yermack D (2017) Corporate governance and blockchains. Rev Finance 21(1):7-31

Yli-Huumo J, Ko D, Choi S, Park S, Smolander K (2016) Where is current research on blockchain technology? A systematic review. PLoS One 11(10):e0163477

Yuan Y, Wang F-Y (2016) Towards blockchain-based intelligent transportation systems. In: 19th IEEE international conference on intelligent transportation systems, Rio de Janeiro. IEEE, pp 2663-2668

Zhang Y, Wen J (2015) An IoT electric business model based on the protocol of bitcoin. In: 18th International conference on intelligence in next generation networks, Paris. IEEE, pp 184-191

Zhang J, Xue N, Huang X (2016) A secure system for pervasive social network-based healthcare. IEEE Access 4:9239-9250

Zhu Y, Guo R, Gan G, Tsai WT (2016) Interactive incontestable signature for transactions confirmation in bitcoin blockchain. In: IEEE 40th annual computer software and applications conference, Atlanta. IEEE, pp 443-448

Zou J, Wang Y, Orgun MA (2016) A dispute arbitration protocol based on a peer-to-peer service contract management scheme. In: IEEE International conference on web services, San Francisco. IEEE, pp 41-48 